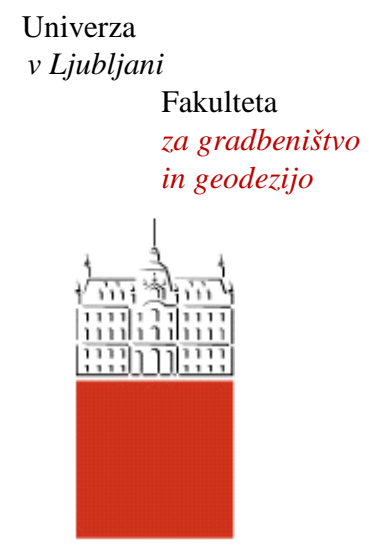

Jamova 2

1000 Ljubljana, Slovenija http://www3.fgg.uni-lj.si//

\section{DRUGG - Digitalni repozitorij UL FGG http://drugg.fgg.uni-lj.si/}

Ta članek je avtorjeva zadnja recenzirana različica, kot je bila sprejeta po opravljeni recenziji.

Prosimo, da se pri navajanju sklicujte na bibliografske podatke, kot je navedeno:
University
of Ljubljana

Faculty of

Civil and Geodetic

Engineering

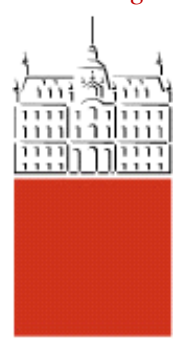

Jamova 2

SI - 1000 Ljubljana, Slovenia

http://www3.fgg.uni-lj.si/

DRUGG - The Digital Repository http://drugg.fgg.uni-lj.si/

This version of the article is author's manuscript as accepted for publishing after the review process.

When citing, please refer to the publisher's bibliographic information as follows:

Brank, B., Briseghella, L., Tonello, N., Damjanić, F. B. 1998. On non-linear dynamics of shells : implementation of energy-momentum conserving algorithm for a finite rotation shell model. International journal for numerical methods in engineering 42, 3: 409-442.

DOI: 10.1002/(SICI)1097-0207(19980615)42:3<409::AID-NME363>3.0.CO;2-B 


\title{
ON NON-LINEAR DYNAMICS OF SHELLS: IMPLEMENTATION OF ENERGY-MOMENTUM CONSERVING ALGORITHM FOR A FINITE ROTATION SHELL MODEL
}

\author{
BOŠTJAN BRANK ${ }^{1,3}$, LAMBERTO BRISEGHELLA $^{2}$, NICOLA TONELL0 ${ }^{2}$ \\ AND FRANO B. DAMJANIĆ ${ }^{3}$ \\ ${ }^{1}$ National Building and Civil Engineering Institute, Dimičeva 12, 1109 Ljubljana, Slovenia \\ 2 Istituto di Scienza e Tecnica delle Costruzioni, University of Padua, Via Marzolo 9, 35100 Padua, Italy \\ 3 Institute of Structural and Earthquake Engineering, Faculty of Civil and Geodetic Engineering, University of \\ Ljubljana, Jamova 2, 1000 Ljubljana, Slovenia
}

\begin{abstract}
Continuum and numerical formulations for non-linear dynamics of thin shells are presented in this work. An elastodynamic shell model is developed from the three-dimensional continuum by employing standard assump- tions of the first-order shear-deformation theories. Motion of the shell-director is described by a singularity- free formulation based on the rotation vector. Temporal discretization is performed by an implicit, one-step, second-order accurate, time-integration scheme. In this work, an energy and momentum conserving algo- rithm, which exactly preserves the fundamental constants of the shell motion and guaranties unconditional algorithmic stability, is used. It may be regarded as a modification of the standard mid-point rule. Spatial discretization is based on the four-noded isoparametric element. Particular attention is devoted to the consis- tent linearization of the weak form of the initial boundary value problem discretized in time and space, in order to achieve a quadratic rate of asymptotic convergence typical for the Newton-Raphson based solution procedures. An unconditionally stable time finite element formulation suitable for the long-term dynamic computations of flexible shell-like structures, which may be undergoing large displacements, large rotations and large motions is therefore obtained. A set of numerical examples is presented to illustrate the present approach and the performance of the isoparametric four-noded shell finite element in conjunction with the implicit energy and momentum conserving timeintegration algorithm.
\end{abstract}

KEY WORDS: non-linear shell dynamics; energy-momentum method; finite elements

\section{INTRODUCTION}

This work is concerned with aspects of non-linear dynamic analysis of flexible shells. Attention is restricted to elastic shells which may be undergoing large displacements, large rotations and large motions. Continuum formulation is developed from the three-dimensional theory by employing standard assumptions on the distribution of the displacement field in the shell boda (e.g. Reference 1) while the treatment of temporal discretization follows Simo et al. $^{2}$ and Simo and Tarnow ${ }^{3}$. 
The non-linear behaviour of thin shells may be reliably presented by the so-called single- or inextensible-director shell models. In this work, we develop a single-director thin-shell model, equivalent to the Cosserat-surface model presented by Simo and Fox, ${ }^{4}$ by approximating the terms describing the shell geometry. Rotation field enters the shell formulation through the basic kinematic assumption. For the dynamic problems it is necessary to perform such local parametrization of rotations that singularities are avoided. Accordingly, the exponential mapping (e.g. Reference 5 ) is used for that purpose.

In situations, when shell is undergoing unconstrained motion governed only by initial conditions, the continuum dynamic formulation conserves two fundamental constants of such motion, namely the total linear and the total angular momentum. If the shell model is specialized by assuming hyperelastic constitutive response and conservative loading, the system becomes Hamiltonian. In that case, the continuum dynamic formulation also conserves the total energy of the system.

The starting point for a dynamic finite element formulation is usually the weak form of the initial boundary value problem. To obtain a fully discrete formulation, the temporal and the spatial discretizations of the weak form need to be subsequently applied. The Newmark family of implicit single-step integration algorithms (e.g. Reference 6) is widely used for the former discretization. However, Newmark's algorithms are unconditionally stable for linear problems, but only conditionally stable for non-linear problems. This has been recently demonstrated for the so-called mid-point and trapezoidal rules for various mechanical systems-rigid bodies, solids, shells and rods-by Simo, Tarnow and co-workers ${ }^{2,3,7-11}$ and Crisfield and Galvanetto. ${ }^{12,13}$

The criterion for the stability of time-integration algorithms in non-linear range is rather related to the conservation of energy. Therefore, the above-mentioned authors proposed time-integration schemes which conserve energy of the Hamiltonian systems. The proposed algorithms demonstrate numerical stability and they also yield accurate solutions for long-term responses. Hence, besides the algorithmic stability, the motivation for energy-conserving algorithms arises also from physical point of view.

Simo and co-workers proposed the so-called energy-momentum method, ${ }^{2,3,7-11}$ which may be regarded as a special form of the standard mid-point rule. This method also conserves the total linear and the total angular momentum of a free body. Recently, Kuhl and Ramm ${ }^{14}$ presented the so-called constraint energy-momentum algorithm, which combines the positive features of energy conserving and numerically dissipative time-integration schemes.

The discretization in time in the present work is closely related to the work of Simo, Tarnow and co-workers. ${ }^{2,3,7-11}$ However, we believe that there are the following different and complementary aspects of the present formulation: (i) The continuum dynamic formulation is derived by using derived approach and has therefore more finite element implementation oriented character. As it is shown, the conservative laws of a shell motion may be obtained in a very simple manner. (ii) The details of the implementation of energy-momentum method for large-rotation shell models, in particular, the linearization aspects, are addressed in detail. (iii) Some aspects related to the finite rotation formulation are simplified with respect to the formulations presented in References 2 and 3. (iv) The performance of energy-momentum method has been tested by some difficult tests.

Spatial discretization in this work is based on a four-noded shell finite element. Isoparametric interpolations of the semi-discrete governing equations are used, since they preserve the conservation properties of the time-stepping algorithm. As shown in Reference 2, the finite element interpolations constructed in a different way, e.g. Reference 1, do not achieve this property. To avoid transverse shear locking, a so-called Assumed Natural Strain (ANS) approach, in the form suggested by Dvorkin and Bathe, ${ }^{15}$ is adopted. 
From the computational point of view, a robust and efficient solution algorithm with high rate of convergence is required. The numerical formulation is therefore characterized by consistent linearization of the fully discrete weak form of the initial boundary value problem. A quadratic rate of asymptotic convergence is achieved.

\section{DYNAMIC THIN SHELL MODEL}

In this section a dynamic shell model is developed from the three-dimensional continuum. For the distribution of the displacement field in the shell body the standard (the simplest possible) assumptions are employed.

\subsection{Basic kinematics of the three-dimensional shell-like body}

Following traditional approach, a shell is treated as a special type of a three-dimensional body embedded in the three-dimensional Euclidean space $\mathbb{R}^{3}$. From the outset, it is assumed that it obeys certain kinematic relations during the deformation process. It is furthermore assumed that the mid-surface of the shell is smooth and its initial thickness is constant.

2.1.1. Preliminaries. In the present work, a shell model is adopted, which basic characteristic is an assumption that any configuration of a shell-like body in $\mathbb{R}^{3}$ can be completely described in terms of two objects: (i) a shell mid-surface and (ii) an independent vector field of unit length, also called a shell-director (vector) field. This form of the shell body description may be obtained by employing standard kinematic assumption of linear distribution of the displacement field through the shell thickness.

Let $\mathscr{B} \subset \mathbb{R}^{3}$ define a reference configuration of the shell-like body. A motion is given by a parameter-dependent mapping $\chi(t): \mathscr{B} \rightarrow \mathbb{R}^{3}$. For some fixed $t \in[0, T] \subset \mathbb{R}^{+}$a mapping $t \rightarrow \chi(\mathscr{B}, t)$ defines a configuration. We use a notation $\chi_{t}(\mathscr{B})=\chi(\mathscr{B}, t)$. A material point, $X$, is identified with its co-ordinates in $\mathbb{R}^{3}$ : It occupies positions $\overline{\mathbf{X}} \in \mathscr{B}$ and $\overline{\mathbf{x}}=\chi_{t}(\overline{\mathbf{X}}) \in \chi_{t}(\mathscr{B})$ at times $t=0$ and $t \in(0, T]$, respectively. In the following, $\mathscr{B}$ and $\mathscr{S}=\chi_{t}(\mathscr{B})$ will be referred to as reference (initial) and current configuration.

We denote a smooth surface in $\mathscr{B}$, referred as a (reference) mid-surface, as $\mathscr{M}_{0}$. Let a shell body be parametrized by a set of curvilinear co-ordinates $\left\{\xi^{1}, \xi^{2}, \xi\right\}$, which are attached to the body. Two co-ordinates, $\xi^{1}$ and $\xi^{2}$, parametrize the mid-surface, while $\xi$ is a parameter in the thickness direction, i.e. $\xi \in[-H / 2, H / 2]=\left[H^{-}, H^{+}\right]$, where $H$ is the reference shell thickness. Reference and current configuration may now be written as

$$
\overline{\mathbf{X}}\left(\xi^{1}, \xi^{2}, \xi\right)=\mathbf{X}\left(\xi^{1}, \xi^{2}\right)+\xi \mathbf{T}\left(\xi^{1}, \xi^{2}\right), \quad \overline{\mathbf{x}}\left(\xi^{1}, \xi^{2}, \xi\right)=\mathbf{x}\left(\xi^{1}, \xi^{2}\right)+\xi \mathbf{t}\left(\xi^{1}, \xi^{2}\right)
$$

respectively, where $\mathbf{X}\left(\xi^{1}, \xi^{2}\right)$ and $\mathbf{x}\left(\xi^{1}, \xi^{2}\right)$ denote mid-surface configurations, while $\mathbf{T}\left(\xi^{1}, \xi^{2}\right)$ and $\mathbf{t}\left(\xi^{1}, \xi^{2}\right)$ denote an inextensible shell-director fields of unit length. The displacement vector of a particular material point may be introduced as $\mathbf{U}=\overline{\mathbf{x}}-\overline{\mathbf{X}}$. This leads to a linear dependance of the displacement vector on $\xi$ co-ordinate

$$
\mathbf{U}=\mathbf{u}+\xi(\mathbf{t}-\mathbf{T})
$$

where $\mathbf{u}$ represents displacement of the related mid-surface point. It is assumed that $\mathbf{T}$ coincides with mid-surface normal. 
In what follows, it proves convenient to employ compact notation in a form $\boldsymbol{\Phi}_{0}: \mathscr{A} \times[-H / 2, H / 2]$ $\rightarrow \mathscr{B}$ and $\boldsymbol{\Phi}_{t}: \mathscr{A} \times[-H / 2, H-2] \rightarrow \mathscr{S}$, where $\mathscr{A} \subset \mathbb{R}^{2}$ is a parametric plane, such that $\left(\xi^{1}, \xi^{2}\right) \in \mathscr{A}$. We use a notation $\boldsymbol{\Phi}_{t}=\boldsymbol{\Phi}$. Clearly, the relation $\chi_{t}=\boldsymbol{\Phi} \circ \boldsymbol{\Phi}_{0}^{-1}$ holds.

Translational velocity vector of the mid-surface point at time $t>0$ is denoted as $\dot{\mathbf{x}}$ and the angular velocity of the shell-director $\mathbf{t}$ is denoted as $\boldsymbol{\omega}$. Since the shell-director is inextensible, we may obtain relation

$$
\omega=\mathbf{t} \times \dot{\mathbf{t}}
$$

through the following steps: $\mathbf{t} \cdot \mathbf{t}=1, \mathbf{t} \cdot \dot{\mathbf{t}}=0, \mathbf{t} \cdot \boldsymbol{\omega}=0, \dot{\mathbf{t}}=\boldsymbol{\omega} \times t$ and $\mathbf{t} \times \dot{\mathbf{t}}=\mathbf{t} \times(\boldsymbol{\omega} \times \mathbf{t})=\boldsymbol{\omega}(\mathbf{t} \cdot \mathbf{t})-$ $\mathbf{t}(\mathbf{t} \cdot \boldsymbol{\omega})=\boldsymbol{\omega}$.

We also use compact notation for velocities: $\dot{\Phi}=(\dot{\mathbf{x}}, \dot{\mathbf{t}})$. Initial velocities at time $t=0$ are denoted as $\dot{\boldsymbol{\Phi}}_{0}=(\dot{\mathbf{X}}, \dot{\mathbf{T}})$.

2.1.2. Motion of the shell director. Motion of an inextensible shell director vector may be described as

$$
\mathbf{t}=\mathbf{R T}
$$

where $\mathbf{R} \in \operatorname{SO}(3)$ is a rotation tensor $\left(\mathbf{R}^{-1}=\mathbf{R}^{\mathrm{T}}\right.$ and $\left.\operatorname{det}[\mathbf{R}]=1\right)$. Any rotation $\mathbf{R}$ is associated with a skew-symmetric tensor $\mathbf{S} \in \operatorname{so}(3)\left(\mathbf{S}=-\mathbf{S}^{\mathrm{T}}\right)$ through an exponential mapping $\mathbf{R}=\exp [\mathbf{S}]$ (see, e.g. Reference 5). Any $\mathbf{S}$ further possesses a so-called axial vector $\mathbf{s} \in \mathbb{R}^{3}$, such that $\mathbf{S s}=0$ and $\mathbf{s} \times \mathbf{b}=\mathbf{S b}$ for every $\mathbf{b} \in \mathbb{R}^{3}$. Axial vector $\mathbf{s}$ is an eigenvector of $\mathbf{R}$ with eigenvalue 1, i.e. $\mathbf{R}$ defines rotation around $\mathbf{s}$. Its norm, $\|\mathbf{s}\|$, defines a magnitude of the rotation.

Many possibilities exist for local parametrization of the rotation tensor. For the dynamical model, it is necessary to choose parametrization without singularities in the range $0 \leqslant\|\mathbf{s}\|<2 \pi$. In this work, we consider explicit formula for exponential mapping, which completely determines rotation with three components of vector $\mathbf{s}$ and its magnitude $\|\mathbf{s}\|$

$$
\mathbf{R}=\exp [\mathbf{S}]=\mathbf{I}+\frac{\sin \|\mathbf{s}\|}{\|\mathbf{s}\|} \mathbf{S}+\frac{1-\cos \|\mathbf{s}\|}{\|\mathbf{s}\|^{2}} \mathbf{S}^{2}
$$

Because of (4) eigenvector $\mathbf{S}$ is perpendicular to $t$ and $\mathbf{T}$, i.e.

$$
\mathbf{t} \cdot \mathbf{s}=0, \quad \mathbf{T} \cdot \mathbf{s}=0
$$

Relations (6) are constrains to rotation (5), and reduce its representation to only two independent components. Details are given below in Sections 2.1.3. and 2.3.4.

2.1.3. Rotational variables. Let $\left\{\mathbf{e}_{i}\right\}_{i=1,3}$ be standard (fixed) orthonormal vector basis in $\mathbb{R}^{3}$. For representation of rotational variables it is convenient to choose different orthonormal and midsurface related basis, e.g. $\left\{\mathbf{E}_{i}\right\}_{i=1,3}$. It is defined at a the mid-surface point by relations $\mathbf{E}_{3}=\mathbf{T}$, $\mathbf{E}_{1} \perp \mathbf{E}_{3}$ and $\mathbf{E}_{2}=\mathbf{E}_{3} \times \mathbf{E}_{1}$. Transformation between mid-surface basis $\left\{\mathbf{E}_{i}\right\}$ and fixed basis $\left\{\mathbf{e}_{i}\right\}$ takes the standard form: $\mathbf{E}_{i}=\boldsymbol{\Lambda}^{0} \mathbf{e}_{i}$, where $\boldsymbol{\Lambda}^{0} \in \mathrm{SO}(3)$ is

$$
\Lambda^{0}=\left[\mathbf{E}_{1}, \mathbf{E}_{2}, \mathbf{T}\right]
$$

Having defined fixed and mid-surface bases, equation (4) may be further elaborated. Let resolve reference shell-director vector $\mathbf{T}$ in fixed basis as $\mathbf{T}=T^{i} \mathbf{e}_{i}$ and in mid-surface basis as $\mathbf{T}=\bar{T}^{i} \mathbf{E}_{i}$. 
We have $T^{i}=\Lambda_{i j}^{0} \bar{T}^{j}$ and $\bar{T}^{i}=\Lambda_{j i}^{0} T^{j}$. By adopting the following notation:

$$
\mathbf{T}=T^{i} \mathbf{e}_{i}, \quad \overline{\mathbf{T}}=\bar{T}^{i} \mathbf{E}_{i}
$$

(note, that $\bar{T}^{1}=\bar{T}^{2}=0$ and $\bar{T}^{3}=1$ ), we may write

$$
\mathbf{T}=\boldsymbol{\Lambda}^{0} \overline{\mathbf{T}}
$$

By inserting (9) into (4) we finally obtain

$$
\mathbf{t}=\mathbf{R} \mathbf{T}=\mathbf{R} \Lambda^{0} \overline{\mathbf{T}}=\boldsymbol{\Lambda} \overline{\mathbf{T}}=\left[\Lambda_{13}, \Lambda_{23}, \Lambda_{33}\right]^{\mathrm{T}}
$$

$\Lambda \in S O(3)$ represents rotational matrix related to the current state. It is clear from (10), (5) and (6) that $\boldsymbol{\Lambda}$ is a two-parametric unknown kinematic variable associated with the through-thickness part of the displacement field (see (2)).

2.1.4. Strain measures. The covariant vector basis at any material point is obtained by standard definition (Greek indices take values 1 to 2 and Latin indices run from 1 to 3 ):

$$
\mathbf{G}_{\alpha}=\frac{\partial \overline{\mathbf{X}}}{\partial \xi^{\alpha}}, \mathbf{G}_{3}=\frac{\partial \overline{\mathbf{X}}}{\partial \xi} \quad \text { and } \quad \mathbf{g}_{\alpha}=\frac{\partial \overline{\mathbf{x}}}{\partial \xi^{\alpha}}, \mathbf{g}_{3}=\frac{\partial \overline{\mathbf{x}}}{\partial \xi}
$$

Here, $\xi^{\alpha}$ are mid-surface convected co-ordinates and $\xi$ is a non-curved parameter in the thickness direction. The dual basis $\mathbf{G}^{\alpha}$ and $\mathbf{G}^{3}$ is defined conventionally by the inner product $\mathbf{G}^{i} \cdot \mathbf{G}_{j}=\delta_{j}^{i}$ where $\delta_{j}^{i}$ is a Kronecker delta. The dual basis $\mathbf{g}^{\alpha}$ and $\mathbf{g}^{3}$ is defined analogously. Vectors $\mathbf{A}_{\alpha}=\left.\mathbf{G}_{\alpha}\right|_{\xi=0}$ span a two-dimensional tangent space to $\mathscr{M}_{0}$ at a point $\mathbf{X}$. Their images at current configuration are $\mathbf{a}_{\alpha}=\left.\mathbf{g}_{\alpha}\right|_{\xi=0}$. For further use we define

$$
G=\operatorname{det}\left[G_{i j}\right], \quad A=\operatorname{det}\left[A_{\alpha \beta}\right]=\operatorname{det}\left[A_{i j}\right], \quad \mu_{0}=\sqrt{\frac{G}{A}}, \quad \mathrm{~d} \mathscr{B}=\mu_{0} \mathrm{~d} \xi \mathrm{d} \mathscr{M}_{0}
$$

where $G_{i j}=\mathbf{G}_{i} \cdot \mathbf{G}_{j}, A_{\alpha \beta}=\mathbf{A}_{\alpha} \cdot \mathbf{A}_{\beta}, A_{i j}=\mathbf{A}_{i} \cdot \mathbf{A}_{j}$. Note, that $\mathbf{A}_{3}=\mathbf{A}^{3}=\mathbf{T}$. The contravariant components of the reference mid-surface metrics are $A^{i j}=\mathbf{A}^{i} \cdot \mathbf{A}^{j}$ with $\mathbf{A}^{i} \cdot \mathbf{A}_{j}=\delta_{j}^{i}$.

For the material description a suitable strain measures are defined by the Green-Lagrange strain tensor

$$
\mathbf{E}=\frac{1}{2}\left(\mathbf{g}_{i} \cdot \mathbf{g}_{j}-\mathbf{G}_{i} \cdot \mathbf{G}_{j}\right) \mathbf{G}^{i} \otimes \mathbf{G}^{j}=E_{i j} \mathbf{G}^{i} \otimes \mathbf{G}^{j}
$$

which may be written as (note that $\mathbf{G}^{3}=\mathbf{G}_{3}=\mathbf{T}$ )

$$
\mathbf{E}=E_{\alpha \beta} \mathbf{G}^{\alpha} \otimes \mathbf{G}^{\beta}+E_{\alpha 3} \mathbf{G}^{\alpha} \otimes \mathbf{T}+E_{3 \alpha} \mathbf{T} \otimes \mathbf{G}^{\alpha}+E_{33} \mathbf{T} \otimes \mathbf{T}
$$

Expressions for $E_{i j}$ follow from (13) and (11); they are functions of $\xi$ co-ordinate

$$
E_{i j}=E_{i j}^{(0)}+\zeta E_{i j}^{(1)}+(\xi)^{2} E_{i j}^{(2)}
$$


Non-zero components of the strain tensor have the following forms:

$$
\begin{aligned}
& E_{\alpha \beta}^{(0)}=\frac{1}{2}\left(\mathbf{A}_{\alpha} \cdot \mathbf{u}_{\beta}+\mathbf{A}_{\beta} \cdot \mathbf{u}_{, \alpha}+\mathbf{u}_{, \alpha} \cdot \mathbf{u}_{\beta}\right) \\
& E_{\alpha 3}^{(0)}=E_{3 \alpha}^{(0)}=\frac{1}{2}\left[\mathbf{A}_{\alpha} \cdot(\mathbf{t}-\mathbf{T})+\mathbf{u}_{, \alpha} \cdot \mathbf{t}\right] \\
& E_{\alpha \beta}^{(1)}=\frac{1}{2}\left[\mathbf{A}_{\alpha} \cdot\left(\mathbf{t}_{\beta}-\mathbf{T},_{\beta}\right)+\mathbf{A}_{\beta} \cdot\left(\mathbf{t}_{, \alpha}-\mathbf{T},_{\alpha}\right)+\mathbf{u}_{\alpha} \cdot \mathbf{t}_{\beta}+\mathbf{u}_{\beta} \cdot \mathbf{t}_{\alpha}\right] \\
& E_{\alpha \beta}^{(2)}=\frac{1}{2}\left(\mathbf{t}_{\alpha} \cdot \mathbf{t}_{\beta}-\mathbf{T}_{,_{\alpha}} \cdot \mathbf{T}_{\beta}\right)
\end{aligned}
$$

where $(0)_{, \alpha}$ denotes partial derivative of $(0)$ with respect to $\xi^{\alpha}$. Components $E_{\alpha \beta}^{(0)}, E_{\alpha 3}^{(0)}$ and $E_{\alpha \beta}^{(1)}$ are conventional shell membrane, transverse shear and bending strains.

The application of the shell theory is reasonable only when some restrictions are imposed on the magnitude of certain fields of the three-dimensional body-such as thickness, curvature or strains. Some of these restrictions are already implicitly assumed by the structure of the basic kinematic assumption itself. However, further estimations of terms defining the strain measures and implicitly the constitutive equations (i.e. stored energy function) are usually performed. In this work we follow a traditional approach (e.g. References 1 and 16) and restrict ourselves to the theory based only on strain components $E_{\alpha \beta}^{(0)}, E_{\alpha 3}^{(0)}$ and $E_{\alpha \beta}^{(1)}$, thus neglecting $E_{\alpha \beta}^{(2)}$. The adopted strain measures are identical to those that may be obtained by the direct or Cosserat surface approach, e.g. Reference 4.

\subsection{Thin shell constitutive equations}

We restrict ourselves to hyperelastic, homogenous and isotropic stored energy function of St. Venant-Kirchhoff type which may be used in the case that Green-Lagrange strains remain rather small. Such situation may be observed in various shell theory applications, even in situations where the body undergoes large displacements accompanied with large rotations. In fact, the small strain situation was implicitly assumed by the structure of the basic kinematic assumption. The second Piola-Kirchhoff stress tensor, $\mathbf{S}=S^{i j} \mathbf{G}_{i} \otimes \mathbf{G}_{i}$, is then $\mathbf{S}=\lambda \operatorname{tr}[\mathbf{E}] \mathbf{I}+2 v \mathbf{E}=\mathbf{H E}$, where $\lambda$ and $v$ are Lamé's material parameters and $\mathrm{H}$ represents fourth-order isotropic tensor with components $\mathrm{H}^{i j k l}=\lambda G^{i j} G^{k l}+v\left(G^{i k} G^{j l}+G^{i l} G^{j k}\right)$.

For the adopted strain measures the stretch in $\xi$ direction, $E_{33}$, equals zero. It is obtained in a standard way by employing an assumption of zero stress in that direction. In the case of small strains we may assume that the component of the second Piola-Kirchhoff stress tensor is vanishing, i.e. $S^{33}=0$. A thin shell hyperelastic constitutive model for small strains emerges by explicit enforcing of that constraint. Accordingly, the three-dimensional constitutive law is condensed, resulting in $\tilde{C}^{i j k l}=\mathrm{H}^{i j k l}-\mathrm{H}^{33 k l} / \mathrm{H}^{3333}, i j \neq 33, k l \neq 33$, where $\tilde{\mathrm{C}}^{i j k l}$ are (rational) functions of $\xi$. The integration of stress tensor components, $S^{i j}=\tilde{\mathrm{C}}^{i j k l} E_{k l}, i j \neq 33, k l \neq 33$, yields the second Piola-Kirchhoff stress resultants of the present model

$$
\begin{aligned}
n^{i j} & =\int_{H^{-}}^{H^{+}} \tilde{\mathrm{C}}^{i j k l}\left(E_{k l}^{(0)}+\xi E_{k l}^{(1)}\right) \mu_{0} \mathrm{~d} \xi, \quad i j \neq 33, k l \neq 33 \\
m^{\alpha \beta} & =\int_{H^{-}}^{H^{+}} \tilde{\mathrm{C}}^{\alpha \beta \gamma \delta}\left(E_{\gamma \delta}^{(0)}+\xi E_{\gamma \delta}^{(1)}\right) \xi \mu_{0} \mathrm{~d} \xi
\end{aligned}
$$

In what follows, we approximate the terms describing the shell geometry at initial configuration: a neglection of the variation of the metrics with respect to the $\xi$ co-ordinate is assumed. We are 
thus able to obtain non-coupled stored energy function and to perform simple analytical throughthickness integration. With $\mathbf{G}_{i}=\mathbf{A}_{i}$ and $\mathbf{G}^{i}=\mathbf{A}^{i}$, which can be justified for thin shells, we have

$$
G_{i j}=A_{i j}, \quad G^{i j}=A^{i j} \quad \text { and } \quad \mu_{0}=\sqrt{G / A}=1
$$

By using (18), the components of the constitutive tensor become independent on $\xi$ (i.e. $\tilde{C}^{i j k l}(\xi) \rightarrow$ $\mathrm{C}^{i j k l}$ ) and equations (17) transform to

$$
n^{i j}=\int_{H^{-}}^{H^{+}} \mathrm{C}^{i j k l} E_{k l}^{(0)} \mathrm{d} \xi, \quad i j \neq 33, k l \neq 33, \quad m^{\alpha \beta}=\int_{H^{-}}^{H^{+}} \mathrm{C}^{\alpha \beta \gamma \delta} E_{\gamma \delta}^{(1)}(\xi)^{2} \mathrm{~d} \xi
$$

In what follows, we will also use the following notation for the symmetric stress resultant tensors

$$
\mathbf{n}=n^{\alpha \beta} \mathbf{A}_{\alpha} \otimes \mathbf{A}_{\beta}, \quad \mathbf{q}=n^{\alpha 3} \mathbf{A}_{\alpha} \otimes \mathbf{T}+n^{3 \alpha} \mathbf{T} \otimes \mathbf{A}_{\alpha}, \quad \mathbf{m}=m^{\alpha \beta} \mathbf{A}_{\alpha} \otimes \mathbf{A}_{\beta}
$$

and for adopted strain tensor (see Section 2.1.4)

$$
\mathbf{E}=\boldsymbol{\varepsilon}+\boldsymbol{\gamma}+\xi \mathbf{K}=E_{\alpha \beta}^{(0)} \mathbf{A}^{\alpha} \otimes \mathbf{A}^{\beta}+\left(E_{\alpha 3}^{(0)} \mathbf{A}^{\alpha} \otimes \mathbf{T}+E_{3 \alpha}^{(0)} \mathbf{T} \otimes \mathbf{A}^{\alpha}\right)+\xi E_{\alpha \beta}^{(1)} \mathbf{A}^{\alpha} \otimes \mathbf{A}^{\beta}
$$

Finally, a two-dimensional stored energy function for the present shell model may be constructed. A shell equivalent to the three-dimensional St. Venant-Kirchhoff stored energy function may be represented as a sum of membrane, transverse shear and bending parts

$$
W\left(E_{i j}\right)=W^{\mathrm{n}}\left(E_{\alpha \beta}^{(0)}\right)+W^{\mathrm{q}}\left(E_{\alpha 3}^{(0)}, E_{3 \alpha}^{(0)}\right)+W^{\mathrm{m}}\left(E_{\alpha \beta}^{(1)}\right)
$$

respectively, where

$$
W^{\mathbf{n}}=\frac{1}{2} E_{\alpha \beta}^{(0)} \mathrm{C}_{0}^{\alpha \beta \gamma \delta} E_{\gamma \delta}^{(0)}, \quad W^{\mathrm{q}}=E_{\alpha 3}^{(0)} \mathrm{C}_{0}^{\alpha 3 \beta 3} E_{\beta 3}^{(0)}, \quad W^{\mathrm{m}}=\frac{1}{2} E_{\alpha \beta}^{(1)} \mathrm{C}_{1}^{\alpha \beta \gamma \delta} E_{\gamma \delta}^{(1)}
$$

and

$$
\mathrm{C}_{J}^{i j k l}=\int_{H^{-}}^{H^{+}} \mathrm{C}^{i j k l}(\xi)^{J} \mathrm{~d} \xi, \quad J=0,1
$$

Remark 2.1. For shells with constant thickness, the transverse shear part of stored energy, $W^{\mathrm{q}}$, is uncoupled with membrane and bending parts, $W^{\mathrm{n}}$ and $W^{\mathrm{m}}$, due to orthogonality of $\mathbf{T}$ and $\mathbf{G}_{\alpha}$. The uncoupling of membrane and bending parts, $W^{\mathrm{n}}$ and $W^{\mathrm{m}}$, is due to neglection of the variation of the metrics with respect to $\xi$ co-ordinate.

\subsection{Weak form of the equations of motion}

In this section the weak form of the equations of motion, which represents the starting point for the numerical formulation, is introduced.

2.3.1. Total linear and angular momentum. Let $\rho_{0}$ and $\rho$ be mass densities at reference and current configuration, respectively. The total linear momentum of the shell is defined as

$$
\mathbf{L}(\dot{\Phi})=\int_{\mathscr{S}} \rho(\dot{\mathbf{x}}+\xi \dot{\mathbf{t}}) \mathrm{d} \mathscr{I}=\int_{\mathscr{B}} \rho_{0}(\dot{\mathbf{x}}+\xi \dot{\mathbf{t}}) \mathrm{d} \mathscr{B}=\int_{\mathscr{M}_{0}}\left[\int_{H^{-}}^{H^{+}} \rho_{0}(\dot{\mathbf{x}}+\xi \dot{\mathbf{t}}) \mu_{0} \mathrm{~d} \xi\right] \mathrm{d} \mathscr{M}_{0}
$$


where the statement of conservation of mass, $\rho \mathrm{d} \mathscr{S}=\rho_{0} \mathrm{~d} \mathscr{B}$, is used. Equations (25) motivate definition of a mid-surface linear momentum, $\mathbf{p}$, and a surface mass density on reference configuration, $A_{\rho_{0}}$

$$
\mathbf{p}=A_{\rho_{0}} \dot{\mathbf{x}}, \quad A_{\rho_{0}}=\int_{H^{-}}^{H^{+}} \rho_{0} \mu_{0} \mathrm{~d} \xi \stackrel{\mu_{0}=1}{=} \rho_{0} H
$$

By using (18), the total linear momentum may be expressed as

$$
\mathbf{L}(\dot{\boldsymbol{\Phi}})=\int_{\mathscr{M}_{0}} \mathbf{p} \mathrm{d} \mathscr{M}_{0}
$$

Analogous to (25), the total angular momentum of the shell relative to the origin of the fixed basis is defined as

$$
\mathbf{J}(\boldsymbol{\Phi}, \dot{\boldsymbol{\Phi}})=\int_{\mathscr{M}_{0}}\left[\int_{H^{-}}^{H^{+}} \rho_{0}(\mathbf{x}+\xi \mathbf{t}) \times(\dot{\mathbf{x}}+\xi \dot{\mathbf{t}}) \mu_{0} \mathrm{~d} \xi\right] \mathrm{d} \mathscr{M}_{0}
$$

This motivates definition of the director momentum, $\pi$, and rotation inertia of reference configuration, $I_{\rho_{0}}$,

$$
\pi=I_{\rho_{0}} \dot{\mathbf{t}}, \quad I_{\rho_{0}}=\int_{H^{-}}^{H^{+}} \rho_{0}(\xi)^{2} \mu_{0} \mathrm{~d} \xi \stackrel{\mu_{0}=1}{=} \rho_{0} \frac{H^{3}}{12}
$$

(Note, that (3) offers an alternative definition for the director momentum, i.e. $\bar{\pi}=\mathbf{t} \times \pi=I_{\rho_{0}} \mathbf{t} \times \dot{\mathbf{t}}=$ $I_{\rho_{0}} \omega$.) The total angular momentum may now be expressed as

$$
\mathbf{J}(\boldsymbol{\Phi}, \dot{\Phi})=\int_{\mathscr{M}_{0}}(\mathbf{x} \times \mathbf{p}+\mathbf{t} \times \pi) \mathrm{d} \mathscr{M}_{0}
$$

Remark 2.2. By noting that $\mathrm{d} \mathscr{S}=\mu \sqrt{a} \mathrm{~d} \xi \mathrm{d} \xi^{1} \mathrm{~d} \xi^{2}$, where $\mu=\sqrt{g / a}, g=\operatorname{det}\left[\mathbf{g}_{i} \cdot \mathbf{g}_{j}\right]$ and $a=\operatorname{det}$ $\left[\mathbf{a}_{\alpha} \cdot \mathbf{a}_{\beta}\right]$, the mid-surface conservation laws may be expressed as $A_{\rho_{0}} \sqrt{A}=A_{\rho} \sqrt{a}$ and $I_{\rho_{0}} \sqrt{A}=I_{\rho} \sqrt{a}$, where $A_{\rho}$ and $I_{\rho}$ are surface mass density and rotation inertia of the current configuration.

2.3.2. Configuration and phase spaces. A collection of all possible configurations of the shell forms the so-called configuration space. Due to the structure of the basic kinematic assumption, it may be defined as

$$
Q=\left\{\boldsymbol{\Phi}=(\mathbf{x}, \mathbf{t}) \mid \mathbf{x}: \mathscr{A} \rightarrow \mathbb{R}^{3}, \mathbf{t}: \mathscr{A} \rightarrow S^{2}\right\}
$$

where $S^{2}=\left\{\mathbf{t} \in \mathbb{R}^{3} \mid\|\mathbf{t}\|=1\right\}$ denotes a unit sphere. The velocities of $\boldsymbol{\Phi}$, denoted as $\dot{\boldsymbol{\Phi}}=(\dot{\mathbf{x}}, \dot{\mathbf{t}})$, are transformed to momenta $\mathbf{p}$ and $\pi$ by $(26)_{1}$ and (29) $)_{1}$.

We will also use a compact notation for momenta: $\Pi=(\mathbf{p}, \pi)$. A phase space for the shell body is defined as $P=\{\mathbf{Z}=(\boldsymbol{\Phi}, \boldsymbol{\Pi})\}$.

2.3.3. Kinetic and potential energies. Dynamic formulation necessitates definition of the kinetic energy. It may be represented as

$$
K(\mathbf{\Pi})=\frac{1}{2} \int_{\mathscr{M}_{0}}\left(A_{\rho_{0}} \dot{\mathbf{x}} \cdot \dot{\mathbf{x}}+I_{\rho_{0}} \dot{\mathbf{t}} \cdot \dot{\mathbf{t}}\right) \mathrm{d} \mathscr{M}_{0}=\frac{1}{2} \int_{\mathscr{M}_{0}}\left(\frac{1}{A_{\rho_{0}}} \mathbf{p} \cdot \mathbf{p}+\frac{1}{I_{\rho_{0}}} \pi \cdot \pi\right) \mathrm{d} \mathscr{M}_{0}
$$


In what follows, we consider the external loading to be dead and conservative. This implies the existence of the potential function of the applied external loads, $V_{\text {ext }}(\Phi)$. The total potential energy of the system is then defined as

$$
V(\boldsymbol{\Phi})=V_{\text {int }}(\boldsymbol{\Phi})+V_{\text {ext }}(\boldsymbol{\Phi})
$$

where $V_{\text {int }}(\Phi)$ denotes the total elastic stored energy and $V_{\text {ext }}(\Phi)$ is the potential energy of the external loads. Internal energy is given as

$$
V_{\text {int }}(\boldsymbol{\Phi})=\int_{\mathscr{M}_{0}}\left(W^{\mathrm{n}}+W^{\mathrm{q}}+W^{\mathrm{m}}\right) \mathrm{d} \mathscr{M}_{0}
$$

with $W^{\mathrm{n}}, W^{\mathrm{q}}$ and $W^{\mathrm{m}}$ defined in (23), while the structure of $V_{\text {ext }}(\Phi)$ will not be discussed here. The total energy of such system, Hamiltonian, is defined as $H(\mathbf{Z})=K(\boldsymbol{\Pi})+V(\boldsymbol{\Phi})$.

2.3.4. Space of test functions. The space of test functions (or admissible variations of a configuration $\Phi$ ), denoted as $T_{\Phi} Q$, is introduced as

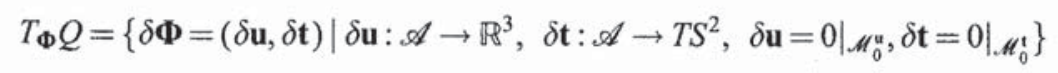

$\mathscr{M}_{0}^{\mathbf{u}}$ and $\mathscr{M}_{0}^{\mathrm{t}}$ are parts of the mid-surface $\mathscr{M}_{0}$ with $\mathbf{u}$ and $\mathbf{t}$ prescribed, and $T S^{2}$ is linear space of vectors tangent to $S^{2}$ at $\mathbf{t}$. Here, $\delta \mathbf{u}$ is an arbitrary test function which represents virtual displacements of the mid-surface and $\delta \mathbf{t}$ is an arbitrary test function which satisfies condition

$$
\delta \mathbf{t} \cdot \mathbf{t}=0
$$

Admissible variations of $\mathbf{Z}$ are denoted as $(\delta \Phi, \delta \Pi)$, where $\delta \Pi=(\delta \mathbf{p}, \delta \pi)$.

Admissible variations of a configuration $\Phi=(\mathbf{x}, \mathbf{t})$ are obtained by a systematic application of the directional derivative to a one-parameter family of configurations $\boldsymbol{\Phi}_{\varepsilon}=\left(\mathbf{x}_{\varepsilon}, \mathbf{t}_{\varepsilon}\right)$. Variations are then obtained by an ordinary differentiation with respect to $\varepsilon$ as $(\delta \mathbf{x}, \delta \mathbf{t})=\left.(\mathrm{d} / \mathrm{d} \varepsilon)\right|_{\varepsilon=0}\left(\mathbf{x}_{\varepsilon}, \mathbf{t}_{\varepsilon}\right)$.

Linear structure of $\mathbb{R}^{3}$ provides a simple expression for the curve $\mathbf{x}_{\varepsilon}$, i.e. $\mathbf{x}_{\varepsilon}=\mathbf{x}+\varepsilon \delta \mathbf{u}$, which yields $\delta \mathbf{x}=\delta \mathbf{u}$. On the other hand, one-parameter curve $\mathbf{t}_{\varepsilon}$ may be obtained in two different ways:

(i) It may be defined as

$$
\mathbf{t}_{\varepsilon}=\mathbf{R}_{\varepsilon} \mathbf{T}=\left[\exp \left(\varepsilon \delta \mathbf{S}_{\mathrm{L}}\right)\right] \mathbf{R} \mathbf{T}=\left[\exp \left(\varepsilon \delta \mathbf{S}_{\mathrm{L}}\right)\right] \mathbf{t}
$$

where $\mathbf{t}_{\varepsilon} \in S^{2}$ and $\delta \mathbf{S}_{\mathrm{L}}$ is a skew-symmetric tensor with corresponding axial vector $\delta \mathbf{s}_{\mathrm{L}}$. Clearly, $\delta \mathbf{s}_{\mathrm{L}} \cdot \mathbf{t}=0$. The differentiation of $\mathbf{t}_{\varepsilon}$ yields

$$
\delta \mathbf{t}=\delta \mathbf{S}_{\mathrm{L}} \mathbf{t}=\delta \mathbf{S}_{\mathrm{L}} \times \mathbf{t}
$$

(ii) Alternatively, $\mathbf{t}_{\varepsilon}$ and $\delta \mathbf{t}$ may be defined as

$$
\mathbf{t}_{\varepsilon}=\mathbf{R}_{\varepsilon} \mathbf{T}=\mathbf{R}\left[\exp \left(\varepsilon \delta \mathbf{S}_{\mathbf{R}}\right)\right] \mathbf{T} \Rightarrow \delta \mathbf{t}=\mathbf{R} \delta \mathbf{S}_{\mathbf{R}} \mathbf{T}=\mathbf{R}\left(\delta \mathbf{s}_{\mathbf{R}} \times \mathbf{T}\right)
$$

where $\delta \mathbf{s}_{\mathrm{R}}$ denotes an axial vector of the skew-symmetric tensor $\delta \mathbf{S}_{\mathrm{R}}$. By introducing notation $\left(\delta \mathbf{s}_{\mathrm{R}} \times \mathbf{T}\right)=\delta \mathbf{T}$, the variation of curve $\mathbf{t}_{\varepsilon}$ may be written as

$$
\delta \mathbf{t}=\mathbf{R} \delta \mathbf{T}
$$

Since $\delta \mathbf{t} \cdot \mathbf{t}=\mathbf{R T} \cdot \mathbf{R} \delta \mathbf{T}=\delta \mathbf{T} \cdot \mathbf{T}=0$ and $\delta \mathbf{s}_{\mathbf{R}} \cdot \mathbf{T}=0$, it follows that vectors $\delta \mathbf{s}_{\mathbf{R}}, \mathbf{T}$ and $\delta \mathbf{T}$ are orthogonal. 
By analogy with (9) the components of $\delta \mathbf{T}$ may be resolved relative to the mid-surface basis as $\delta \mathbf{T}=\delta \bar{T}^{i} \mathbf{E}_{i}$ and relative to the fixed basis as $\delta \mathbf{T}=\delta T^{i} \mathbf{e}_{i}$. We have $\delta T^{i}=\Lambda_{i j}^{0} \delta \bar{T}^{j}$. By introducing notation $\delta \overline{\mathbf{T}}=\left[\delta \bar{T}^{1}, \delta \bar{T}^{2}, 0\right]^{\mathrm{T}}$ (note, that $\overline{\mathbf{T}} \cdot \delta \overline{\mathbf{T}}=0$, see Section 2.1.2), equation (40) may be rewritten as

$$
\delta \mathbf{t}=\mathbf{R} \mathbf{\Lambda}^{0} \delta \overline{\mathbf{T}}=\mathbf{\Lambda} \delta \overline{\mathbf{T}}
$$

Since the third component of $\delta \overline{\mathbf{T}}$ is zero, equation (41) may be further elaborated. By introducing a $(3 \times 2)$ matrix $\bar{\Lambda}$ obtained simply by dropping the third column of $\Lambda$, and a two-dimensional vector $\delta \widehat{\mathbf{T}}=\left[\delta \bar{T}^{1}, \delta \bar{T}^{2}\right]^{\mathrm{T}}$, we finally have

$$
\delta \mathbf{t}=\overline{\mathbf{\Lambda}} \delta \widehat{\mathbf{T}}
$$

2.3.5. Formulation of the weak form of the equations of motion. By defining the kinetic and potential energies, the Lagrangian differential equations of motion may be transformed to the classical Hamiltonian canonical equations subjected to configuration and momenta initial conditions (e.g. Reference 17). These equations may be written in the weak form as

$$
\int_{\mathscr{M}_{0}} \dot{\boldsymbol{\Pi}} \cdot \delta \boldsymbol{\Phi} \mathrm{d} \mathscr{M}_{0}+\frac{\partial H(\mathbf{Z})}{\partial \boldsymbol{\Phi}} \cdot \delta \boldsymbol{\Phi}=0, \quad \int_{\mathscr{M}_{0}} \dot{\boldsymbol{\Phi}} \cdot \delta \boldsymbol{\Pi} \mathrm{d} \mathscr{M}_{0}-\frac{\partial H(\mathbf{Z})}{\partial \boldsymbol{\Pi}} \cdot \delta \boldsymbol{\Pi}=0
$$

where $H(\mathbf{Z})$ is a sum of kinetic and potential energies of the shell, defined by (32) and (33). From $(43)_{1}$ the weak form of momentum equations may be reproduced

$$
G_{\text {dyn }}(\dot{\Pi}, \boldsymbol{\Phi}, \delta \boldsymbol{\Phi})=G_{\text {ine }}(\dot{\Pi}, \delta \boldsymbol{\Phi})+G_{\text {stat }}(\boldsymbol{\Phi}, \delta \Phi)-G_{\text {ext }}(\delta \Phi)=0
$$

$G_{\text {ine }}(\dot{\boldsymbol{\Pi}}, \delta \Phi)$, which is due to inertial forces, and the part denoted as $G_{\text {stat }}(\boldsymbol{\Phi}, \delta \Phi)$, which has the same form as in non-linear static formulation, are defined as

$$
\begin{aligned}
G_{\text {ine }}(\dot{\boldsymbol{\Pi}}, \delta \Phi) & =\int_{\mathscr{M}_{0}}(\dot{\mathbf{p}} \cdot \delta \mathbf{u}+\dot{\pi} \cdot \delta \mathbf{t}) \mathrm{d} \mathscr{M}_{0} \\
G_{\text {stat }}(\boldsymbol{\Phi}, \delta \boldsymbol{\Phi}) & =\int_{\mathscr{M}_{0}}(\mathbf{n}: \delta \varepsilon+\mathbf{q}: \delta \gamma+\mathbf{m}: \delta \mathbf{\kappa}) \mathrm{d} \mathscr{M}_{0}
\end{aligned}
$$

Here, ' $\because$ ' denotes the inner product of two tensors. Stress and strain tensors are defined by $(20)$ and (21). The part due to the external loading is given as $G_{\text {ext }}(\delta \Phi)=-V_{\text {ext }}(\delta \Phi)$. Equation (44) is supplemented by the configuration and momenta initial conditions

$$
\left.(\mathbf{x}, \mathbf{t})\right|_{t=0}=(\mathbf{X}, \mathbf{T}),\left.\quad(\mathbf{p}, \pi)\right|_{t=0}=\left(\mathbf{p}_{0}, \pi_{0}\right)
$$

The strong form of $(43)_{2}$ gives $(26)_{1}$ and $(29)_{1}$.

Strain measure variations, which are obtained by taking the directional derivative of $\mathbf{E}$ in the direction of ( $\delta \mathbf{u}, \delta \mathbf{t})$, may be expressed in the component form as (note, that $\mathbf{a}_{\alpha}=\mathbf{A}_{\alpha}+\mathbf{u}_{, \alpha}$ )

$$
\begin{aligned}
& \delta E_{\alpha \beta}^{(0)}=\frac{1}{2}\left(\mathbf{a}_{\alpha} \cdot \delta \mathbf{u},_{\beta}+\mathbf{a}_{\beta} \cdot \delta \mathbf{u}_{\alpha \alpha}\right) \\
& \delta E_{\alpha 3}^{(0)}=\delta E_{3 \alpha}^{(0)}=\frac{1}{2}\left(\mathbf{a}_{\alpha} \cdot \delta \mathbf{t}+\delta \mathbf{u}_{\alpha} \cdot \mathbf{t}\right) \\
& \delta E_{\alpha \beta}^{(1)}=\frac{1}{2}\left(\mathbf{a}_{\alpha} \cdot \delta \mathbf{t}_{\beta}+\mathbf{a}_{\beta} \cdot \delta \mathbf{t}, \alpha+\delta \mathbf{u}_{, \alpha} \cdot \mathbf{t}, \beta+\delta \mathbf{u}_{\beta} \cdot \mathbf{t},_{\alpha}\right)
\end{aligned}
$$

Note, that equations (47) are linear on $\mathbf{u}$ and $\mathbf{t}$. 
2.3.6. Conservation laws of the shell motion. In this section it is shown that the present continuum formulation conserves the fundamental constants of the shell motion, namely the total energy and the total linear and angular momentum.

The conservation law of the total energy, $\mathrm{d} H(\mathbf{Z}) / \mathrm{d} t=0$, may be obtained by using the test functions in a form $(\dot{\mathbf{x}}, \dot{\mathbf{t}})$ in (44) and by observing that $\mathrm{d} H(\mathbf{Z}) / \mathrm{d} t=\mathrm{d} K(\mathbf{I I}) / \mathrm{d} t+\mathrm{d} V(\boldsymbol{\Phi}) / \mathrm{d} t=$ $G_{\text {dyn }}(\dot{\mathbf{I}}, \boldsymbol{\Phi}, \dot{\boldsymbol{\Phi}})=0$ when $G_{\text {ext }}(\delta \Phi)=0$.

Conservation laws of the total linear and angular momentum may be obtained in a following way. Let us assume, that the motion of the shell is governed only by initial conditions, and is not restricted by displacement/rotation boundary conditions. In that case, the total linear and angular momentum of the shell are conserved in the sense that $\mathrm{d} \mathbf{L} / \mathrm{d} t=\mathbf{0}$ and $\mathrm{d} \mathbf{J} / \mathrm{d} t=\mathbf{0}$. For the present model this can be proven by choosing $(\mathbf{b}, \mathbf{0})$ and $(\mathbf{b} \times \mathbf{x}, \mathbf{b} \times \mathbf{t})$, with $\mathbf{b} \in \mathbb{R}^{3}$, for test functions. By inserting these test functions into (47), we find out that $\delta E_{\alpha \beta}^{(0)}=\delta E_{\alpha 3}^{(0)}=\delta E_{\alpha \beta}^{(1)}=0$. This implies that $G_{\text {stat }}(\boldsymbol{\Phi}, \mathbf{0})=0$ and $G_{\text {stat }}(\boldsymbol{\Phi}, \mathbf{b} \times \mathbf{t})=0$. Hence, the only non-zero part in (44) is the one related to the inertial forces. From $G_{\text {ine }}(\dot{\boldsymbol{\Pi}}, \mathbf{0})=0$ and $G_{\text {ine }}(\dot{\boldsymbol{I}}, \mathbf{b} \times \mathbf{t})=0$ we may finally arrive to the expressions $\mathbf{b} \cdot \dot{\mathbf{L}}=0$ and $\mathbf{b} \cdot \dot{\mathbf{J}}=0$, respectively. Since $\mathbf{b}$ is arbitrary, it follows $\dot{\mathbf{L}}=\dot{\mathbf{J}}=0$.

\section{TIME DISCRETE FORMULATION}

In this section an implicit one-step time-integration scheme is considered. In particular, energymomentum method proposed in Reference 3 is employed. Its construction may be divided into two steps for non-linear elastodynamics: First, an algorithm with momentum conservation is selected, and in the second step an algorithmic approximation of the constitutive equations is constructed in such a way that conservation of the total energy is achieved for the Hamiltonian systems.

\subsection{Update relations for configuration and momenta}

Let a time interval of interest be partitioned into $N$ subintervals as $[0, T]=\bigcup_{n=0}^{N}\left[t_{n}, t_{n+1}\right]$. Considering a typical time subinterval, an algorithmic problem may be stated as: Assuming that initial conditions at $t_{n}$ are given, define algorithmic approximation of configuration, velocities and accelerations at $t_{n+1}$.

For the further use we approximate configuration and associated momenta at $t_{n+1 / 2}, \boldsymbol{\Phi}_{n+1 / 2}$ and $\Pi_{n+1 / 2}$, as linear combination of initial and final values of the time subinterval

$$
\begin{aligned}
& \left(\mathbf{x}_{n+1 / 2}, \mathbf{t}_{n+1 / 2}\right)=\frac{1}{2}\left(\mathbf{x}_{n+1}, \mathbf{t}_{n+1}\right)+\frac{1}{2}\left(\mathbf{x}_{n}, \mathbf{t}_{n}\right) \\
& \left(\mathbf{p}_{n+1 / 2}, \pi_{n+1 / 2}\right)=\frac{1}{2}\left(\mathbf{p}_{n+1}, \pi_{n+1}\right)+\frac{1}{2}\left(\mathbf{p}_{n}, \pi_{n}\right)
\end{aligned}
$$

The differential equations $(26)_{1}$ and $(29)_{1}$ are approximated as

$$
\mathbf{p}_{n+1 / 2}=A_{\rho_{0}} \frac{\mathbf{x}_{n+1}-\mathbf{x}_{n}}{\Delta t} \text { and } \pi_{n+1 / 2}=I_{\rho_{0}} \frac{\mathbf{t}_{n+1}-\mathbf{t}_{n}}{\Delta t}
$$

where $\Delta t=t_{n+1}-t_{n}$ is corresponding time-step size. By using (49) in (48) 2 the momenta at the end of the time step may be written as a function of $\boldsymbol{\Phi}_{n+1}$

$$
\left(\mathbf{p}_{n+1}, \pi_{n+1}\right)=\left(2 A_{\rho_{0}} \frac{\mathbf{x}_{n+1}-\mathbf{x}_{n}}{\Delta t}, 2 I_{\rho_{0}} \frac{\mathbf{t}_{n+1}-\mathbf{t}_{n}}{\Delta t}\right)-\left(\mathbf{p}_{n}, \pi_{n}\right)
$$




\subsection{Time discrete equations of motion}

The time discrete version of (44), evaluated at the intermediate configuration $t_{n+1 / 2}$, may be written as

$$
\begin{aligned}
& \frac{1}{\Delta t} \int_{\mathscr{M}_{0}}\left[\delta \mathbf{u} \cdot\left(\mathbf{p}_{n+1}-\mathbf{p}_{n}\right)+\delta \mathbf{t} \cdot\left(\pi_{n+1}-\pi_{n}\right)\right] \mathrm{d} \mathscr{M}_{0} \\
& \quad=-\left[G_{\text {stat }}\left(\boldsymbol{\Phi}_{n+1 / 2}, \delta \boldsymbol{\Phi}\right)-G_{\text {ext }}(\delta \boldsymbol{\Phi})\right]
\end{aligned}
$$

where equation (51) includes approximation of accelerations at the intermediate configuration

$$
\dot{\mathbf{p}}_{n+1 / 2}=\frac{\mathbf{p}_{n+1}-\mathbf{p}_{n}}{\Delta t}, \quad \dot{\pi}_{n+1 / 2}=\frac{\pi_{n+1}-\pi_{n}}{\Delta t}
$$

By inserting (50) into the left-hand side of (51), the following expression is obtained:

$$
\begin{aligned}
& G_{\text {ine }}\left(\boldsymbol{\Phi}_{n}, \boldsymbol{\Pi}_{n}, \boldsymbol{\Phi}_{n+1}, \delta \boldsymbol{\Phi}\right) \\
& \quad=\frac{2}{\Delta t} \int_{\mathscr{M}_{0}}\left[\left(\frac{A_{\rho_{0}}}{\Delta t}\left(\mathbf{x}_{n+1}-\mathbf{x}_{n}\right)-\mathbf{p}_{n}\right) \cdot \delta \mathbf{u}+\left(\frac{I_{\rho_{0}}}{\Delta t}\left(\mathbf{t}_{n+1}-\mathbf{t}_{n}\right)-\pi_{n}\right) \cdot \delta \mathbf{t}\right] \mathrm{d} \mathscr{M}_{0}
\end{aligned}
$$

To evaluate the right-hand side of $(51)$ at $t_{n+1 / 2}$, relations $(50)$ and $(48)_{1}$ are used. We have

$$
G_{\text {stat }}\left(\boldsymbol{\Phi}_{n}, \boldsymbol{\Phi}_{n+1}, \delta \boldsymbol{\Phi}\right)=\int_{\mathscr{M}_{0}}(\mathbf{n}: \delta \varepsilon+\mathbf{q}: \delta \gamma+\mathbf{m}: \delta \mathbf{\kappa}) \mathrm{d} \mathscr{M}_{0}
$$

where $\delta \boldsymbol{\varepsilon}, \delta \gamma$ and $\delta \mathbf{K}$ are variations of Green-Lagrange strain tensors associated with configuration $\boldsymbol{\Phi}_{n+1 / 2}$. The algorithmic stress resultant tensors $\mathbf{n}, \mathbf{q}$ and $\mathbf{m}$ are not yet defined at this stage.

3.2.1. Variation of strain measures of intermediate configuration. Variations of Green-Lagrange strain tensors at intermediate configuration $\boldsymbol{\Phi}_{n+1 / 2}$ follow from using $(48)_{1}$ in (47). The resulting equations may be additively split into two parts

$$
\begin{aligned}
\delta E_{\alpha \beta}^{(0)}= & \frac{1}{2}\left[\frac{1}{2}\left(\mathbf{x}_{n, \alpha} \cdot \delta \mathbf{u}_{\beta}+\mathbf{x}_{n, \beta} \cdot \delta \mathbf{u}_{\alpha}\right)\right]+\frac{1}{2}\left[\frac{1}{2}\left(\mathbf{x}_{n+1}, \alpha \cdot \delta \mathbf{u}_{\beta}+\mathbf{x}_{n+1, \beta} \cdot \delta \mathbf{u}_{, \alpha}\right)\right] \\
2 \delta E_{\alpha 3}^{(0)}= & \frac{1}{2}\left(\mathbf{x}_{n, \alpha} \cdot \delta \mathbf{t}+\delta \mathbf{u}_{, \alpha} \cdot \mathbf{t}_{n}\right)+\frac{1}{2}\left(\mathbf{x}_{n+1, \alpha} \cdot \delta \mathbf{t}+\delta \mathbf{u},_{\alpha} \cdot \mathbf{t}_{n+1}\right) \\
\delta E_{\alpha \beta}^{(1)}= & \frac{1}{2}\left[\frac{1}{2}\left(\mathbf{x}_{n, \alpha} \cdot \delta \mathbf{t},_{\beta}+\mathbf{x}_{n, \beta} \cdot \delta \mathbf{t}_{, \alpha}+\delta \mathbf{u}_{, \alpha} \cdot \mathbf{t}_{n, \beta}+\delta \mathbf{u}_{, \beta} \cdot \mathbf{t}_{n, \alpha}\right)\right] \\
& +\frac{1}{2}\left[\frac{1}{2}\left(\mathbf{x}_{n+1, \alpha} \cdot \delta \mathbf{t}_{\beta}+\mathbf{x}_{n+1, \beta} \cdot \delta \mathbf{t},_{\alpha}+\delta \mathbf{u}_{\alpha} \cdot \mathbf{t}_{n+1, \beta}+\delta \mathbf{u}_{, \beta} \cdot \mathbf{t}_{n+1, \alpha}\right)\right]
\end{aligned}
$$

Algorithmic approximation of constraint (36) has now a form $\delta \mathbf{t} \cdot \mathbf{t}_{n+1 / 2}=0$. According to (41), we have

$$
\delta \mathbf{t}=\boldsymbol{\Lambda}_{n+1 / 2} \delta \overline{\mathbf{T}}
$$

Rotation $\boldsymbol{\Lambda}_{n+1 / 2}$ rotates vector $\overline{\mathbf{T}}=[0,0,1]^{\mathrm{T}}$ into $\mathbf{t}_{n+1 / 2} /\left\|\mathbf{t}_{n+1 / 2}\right\|$, i.e. $\mathbf{t}_{n+1 / 2} /\left\|\mathbf{t}_{n+1 / 2}\right\|=\boldsymbol{\Lambda}_{n+1 / 2} \mathbf{T}$. Algorithmic approximation of $\delta \mathbf{t} \cdot \mathbf{t}=0$ is then satisfied identically, since $\delta \mathbf{t} \cdot \mathbf{t}_{n+1 / 2}=\delta \overline{\mathbf{T}} \cdot \overline{\mathbf{T}}\left\|\mathbf{t}_{n+1 / 2}\right\|=0$.

\subsection{Conservation properties of the time-stepping algorithm}

The structure of the algorithmic stress resultant tensors, $\mathbf{n}, \mathbf{q}$ and $\mathbf{m}$, has not been discussed so far. In what follows, the stress resultants will be designed in such a way that the total energy of 
the system is exactly preserved within the time step. Under the assumption of dead loading, the energy of the Hamiltonian system is preserved in the time interval $\left[t_{n}, t_{n+1}\right]$ if $H_{n+1}=H_{n}$. It can be shown, that this can be achieved only with the following form of the algorithmic stress resultants

$$
\begin{aligned}
& n^{\alpha \beta}=\frac{1}{2} \mathrm{C}_{0}^{\alpha \beta \gamma \delta}\left[\left(E_{\gamma \delta}^{(0)}\right)_{n+1}+\left(E_{\gamma \delta}^{(0)}\right)_{n}\right] \\
& q^{\alpha 3}=\mathrm{C}_{0}^{\alpha 3 \gamma 3}\left[\left(2 E_{\gamma 3}^{(0)}\right)_{n+1}+\left(2 E_{\gamma 3}^{(0)}\right)_{n}\right], \quad m^{\alpha \beta}=\frac{1}{2} \mathrm{C}_{1}^{\alpha \beta \gamma \delta}\left[\left(E_{\gamma \delta}^{(1)}\right)_{n+1}+\left(E_{\gamma \delta}^{(1)}\right)_{n}\right]
\end{aligned}
$$

Algorithmic strains, used when defining the energy conserving form of algorithmic stress resultants, are, therefore, for the quadratic stored energy function, evaluated as an average of strains at time instants $t_{n}$ and $t_{n+1}$.

To prove that the total linear and angular momentum are conserved by the above-defined time-stepping procedure within the time step, the observations similar to those in Section 2.3.6 may be used. By choosing (b,0) and $\left(\mathbf{b} \times \mathbf{x}_{n+1 / 2}, \mathbf{b} \times \mathbf{t}_{n+1 / 2}\right)$ as test functions, where $\mathbf{b} \in \mathbb{R}^{3}$, we may obtain from (55) the following results: $\delta E_{\alpha \beta}^{(0)}=\delta E_{\alpha 3}^{(0)}=\delta E_{\alpha \beta}^{(1)}=0$. As a consequence we have: $G_{\text {stat }}\left(\boldsymbol{\Phi}_{n+1 / 2}, \mathbf{0}\right)=0$ and $G_{\text {stat }}\left(\boldsymbol{\Phi}_{n+1 / 2}, \mathbf{b} \times \mathbf{t}_{n+1 / 2}\right)=0$. From $G_{\text {ine }}\left(\boldsymbol{\Phi}_{n}, \boldsymbol{\Pi}_{n}, \boldsymbol{\Phi}_{n+1}, \mathbf{0}\right)=0$ and $G_{\text {ine }}\left(\boldsymbol{\Phi}_{n}, \boldsymbol{\Pi}_{n}, \boldsymbol{\Phi}_{n+1}, \boldsymbol{\xi} \times \mathbf{t}_{n+1 / 2}\right)=0$, it then follows that $\mathbf{b} \cdot\left(\mathbf{L}_{n+1}-\mathbf{L}_{n}\right) 1 / \Delta t=0$ and $\mathbf{b} \cdot\left(\mathbf{J}_{n+1}-\mathbf{J}_{n}\right)$ $1 / \Delta t=0$, respectively. Since b is arbitrary, we have $\mathbf{L}_{n+1}=\mathbf{L}_{n}$ and $\mathbf{J}_{n+1}=\mathbf{J}_{n}$.

\subsection{Time discretized matrix formulation}

In advance of the time finite element formulation, the semi-discretized weak form of the equations of motion is presented in the matrix notation. ${ }^{\dagger}$ For that purpose the kinematic and force variables are expressed in the matrix form.

The matrix notation for the vector-valued quantities at time instant $t_{n+1}$ such as displacements, rotational parameters and mid-surface linear and angular momentum is obvious. In the compact form we can write

$$
\boldsymbol{\Phi}_{n+1}=[\mathbf{u}, \mathbf{t}]_{n+1}^{\mathrm{T}}, \quad \boldsymbol{\Pi}_{n+1}=[\mathbf{p}, \boldsymbol{\pi}]_{n+1}^{\mathrm{T}}
$$

The strains and the algorithmic stress resultants may be written in matrix notation as

$$
\begin{aligned}
\boldsymbol{\varepsilon}_{n+1} & =\left[E_{11}^{(0)}, E_{22}^{(0)}, 2 E_{12}^{(0)}\right]_{n+1}^{\mathrm{T}}, \quad \gamma_{n+1}=\left[2 E_{13}^{(0)}, 2 E_{23}^{(0)}\right]_{n+1}^{\mathrm{T}} \\
\boldsymbol{\kappa}_{n+1} & =\left[E_{11}^{(1)}, E_{22}^{(1)}, 2 E_{12}^{(1)}\right]_{n+1}^{\mathrm{T}} \\
\mathbf{n} & =\left[n^{11}, n^{22}, n^{12}\right]^{\mathrm{T}}, \quad \mathbf{q}=\left[q^{13}, q^{23}\right]^{\mathrm{T}}, \quad \mathbf{m}=\left[m^{11}, m^{22}, m^{12}\right]^{\mathrm{T}}
\end{aligned}
$$

with vectors $\mathbf{n}, \mathbf{q}$ and $\mathbf{m}$ given as

$$
\mathbf{n}=\mathrm{C}^{\mathbf{n}} \frac{1}{2}\left(\varepsilon_{n}+\varepsilon_{n+1}\right), \quad \mathbf{q}=\mathrm{C}^{\mathbf{q}} \frac{1}{2}\left(\gamma_{n}+\gamma_{n+1}\right), \quad \mathbf{m}=\mathrm{C}^{\mathbf{m}} \frac{1}{2}\left(\boldsymbol{\kappa}_{n}+\boldsymbol{\kappa}_{n+1}\right)
$$

Here, matrices $C^{n}, C^{m}$ and $C^{q}$ define constitutive relations for membrane, bending and transverse shear parts

$$
\mathrm{C}^{\mathrm{n}}=\frac{E H}{1-v^{2}} \overline{\mathrm{C}}, \quad \mathrm{C}^{\mathrm{m}}=\frac{E H^{3}}{12\left(1-v^{2}\right)} \overline{\mathrm{C}}, \quad \mathrm{C}^{\mathrm{q}}=\kappa G H\left[\begin{array}{c}
A^{11} A^{12} \\
\operatorname{sym} \cdot A^{22}
\end{array}\right]
$$

${ }^{\dagger}$ In the following $\varepsilon, \gamma, \mathbf{k}, \mathbf{n}, \mathbf{q}, \mathbf{m}$ and $\mathbf{E}$ will denote the corresponding vectors while in previous sections the same notation was related to the corresponding tensors 
where, $E, v, G$ and $\kappa$ are elastic modulus, Poisson's ratio, shear modulus $(G=E /[2(1+v)])$ and shear correction factor, respectively, and matrix $\bar{C}$ is

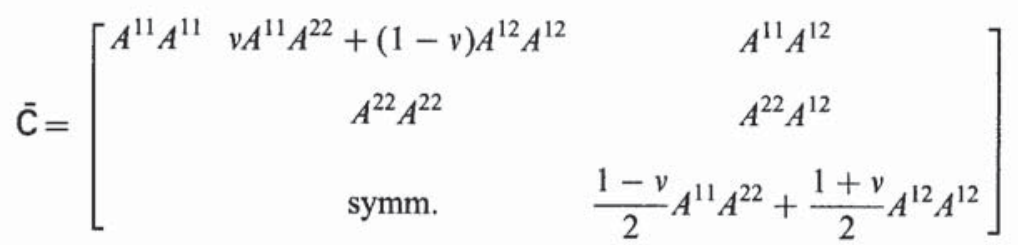

A compact notations for force and strain vectors and constitutive relations read as

$$
\mathbf{r}=[\mathbf{n}, \mathbf{m}, \mathbf{q}]^{\mathrm{T}}, \quad \mathbf{E}=[\varepsilon, \boldsymbol{\kappa}, \boldsymbol{\gamma}]^{\mathrm{T}}, \quad \mathbf{C}=\operatorname{diag}\left[\mathrm{C}^{\mathbf{n}}, \mathrm{C}^{\mathbf{m}}, \mathrm{C}^{\mathrm{q}}\right]
$$

Following standard practice, the generalised strain-displacement operators are introduced in the matrix form as

$$
\begin{aligned}
& \mathbf{B}_{n+1}^{e}=\left[\begin{array}{cc}
\left(\mathbf{x}, \xi^{\xi^{1}}\right)^{\mathrm{T}} \frac{\partial}{\partial \xi^{1}} & \mathbf{0} \\
\left(\mathbf{x}, \xi^{2}\right)^{\mathrm{T}} \frac{\partial}{\partial \xi^{2}} & \mathbf{0} \\
\left(\mathbf{x}, \xi^{2}\right)^{\mathrm{T}} \frac{\partial}{\partial \xi^{1}}+\left(\mathbf{x}, \xi^{1}\right)^{\mathrm{T}} \frac{\partial}{\partial \xi^{2}} & \mathbf{0}
\end{array}\right]_{n+1}, \quad \mathbf{B}_{n+1}^{\mathrm{\gamma}}=\left[\begin{array}{c}
\mathbf{t}^{\mathrm{T}} \frac{\partial}{\partial \xi^{1}}\left(\mathbf{x}, \xi^{1}\right)^{\mathrm{T}} \\
\mathbf{t}^{\mathrm{T}} \frac{\partial}{\partial \xi^{2}}\left(\mathbf{x}, \xi_{\xi^{2}}\right)^{\mathrm{T}}
\end{array}\right]_{n+1} \\
& \mathbf{B}_{n+1}^{\boldsymbol{x}}=\left[\begin{array}{cc}
\left(\mathbf{t}, \xi^{1}\right)^{\mathrm{T}} \frac{\partial}{\partial \xi^{1}} & \left(\mathbf{x}, \xi^{1}\right)^{\mathrm{T}} \frac{\partial}{\partial \xi^{1}} \\
\left(\mathbf{t}, \xi^{2}\right)^{\mathrm{T}} \frac{\partial}{\partial \xi^{2}} & \left(\mathbf{x}, \xi^{2}\right)^{\mathrm{T}} \frac{\partial}{\partial \xi^{2}} \\
\left(\mathbf{t}, \xi^{1}\right)^{\mathrm{T}} \frac{\partial}{\partial \xi^{2}}+\left(\mathbf{t}, \xi^{2}\right)^{\mathrm{T}} \frac{\partial}{\partial \xi^{1}} & \left(\mathbf{x}, \xi^{1}\right)^{\mathrm{T}} \frac{\partial}{\partial \xi^{2}}+\left(\mathbf{x}, \xi^{2}\right)^{\mathrm{T}} \frac{\partial}{\partial \xi^{1}}
\end{array}\right]_{n+1}
\end{aligned}
$$

The membrane transverse shear and bending strain variations at $t_{n+1 / 2},(55)$, may be expressed in a compact form as

$$
\delta \varepsilon=\mathbf{B}_{n+1 / 2}^{\varepsilon} \delta \Phi, \quad \delta \gamma=\mathbf{B}_{n+1 / 2}^{\gamma} \delta \Phi, \quad \delta \mathbf{\kappa}=\mathbf{B}_{n+1 / 2}^{\boldsymbol{\kappa}} \delta \boldsymbol{\Phi}
$$

where the notation $\delta \boldsymbol{\Phi}=[\delta \mathbf{u}, \delta \mathbf{t}]^{\mathrm{T}}$ is used. Note that $\delta \mathbf{t}=\boldsymbol{\Lambda}_{n+1 / 2} \delta \overline{\mathbf{T}}$ (see (56)). Construction of matrix $\boldsymbol{\Lambda}_{n+1 / 2}$ is provided in Box 1. Equations (66) may be rewritten as

$$
\delta \mathbf{E}=\mathbf{B}_{n+1 / 2} \delta \boldsymbol{\Phi}, \quad \text { where } \delta \mathbf{E}=\left\{\begin{array}{c}
\delta \boldsymbol{\varepsilon} \\
\delta \mathbf{\kappa} \\
\delta \gamma
\end{array}\right\}, \quad \mathbf{B}_{n+1 / 2}=\left[\begin{array}{c}
\mathbf{B}_{n+1 / 2}^{\varepsilon} \\
\mathbf{B}_{n+1 / 2}^{\boldsymbol{\kappa}} \\
\mathbf{B}_{n+1 / 2}^{\gamma}
\end{array}\right]
$$

Due to the linearity of (55) on $\mathbf{u}$ and $\mathbf{t}$, it may be easily observed that $\mathbf{B}_{n+1 / 2}=\frac{1}{2}\left[\mathbf{B}_{n}+\mathbf{B}_{n+1}\right]$.

Finally, the inertial and static parts of the weak form of the semi-discretized equation of motion may be written as

$$
G_{\text {ine }}\left(\boldsymbol{\Phi}_{n}, \boldsymbol{\Phi}_{n+1}, \delta \boldsymbol{\Phi}\right)=\frac{1}{\Delta t} \int_{\mathscr{M}_{0}} \delta \boldsymbol{\Phi}^{\mathrm{T}}\left[\begin{array}{ll}
\mathbf{p}_{n+1}-\mathbf{p}_{n} & \mathbf{0} \\
\mathbf{0} & \pi_{n+1}-\pi_{n}
\end{array}\right] \mathrm{d} \mathscr{M}_{0}
$$


Box 1. Evaluation of rotational matrix at intermediate configuration

(i) Known: $\boldsymbol{\Lambda}_{n}, \boldsymbol{\Lambda}_{n+1}, \mathbf{t}_{n}, \mathbf{t}_{n+1}$

(ii) Evaluate:

$$
\begin{aligned}
& a=\cos (\alpha)=\frac{\mathbf{t}_{n} \cdot \mathbf{t}_{n+1}}{\left\|\mathbf{t}_{n}\right\|\left\|\mathbf{t}_{n+1}\right\|} \\
& \mathbf{b}=\mathbf{t}_{n} \times \mathbf{t}_{n+1}
\end{aligned}
$$

(iii) Evaluate rotational matrix at intermediate configuration

$$
\begin{gathered}
\text { IF }(\|1-a\|<\text { TOLER OR }\|\mathbf{b}\|<\text { TOLER OR } \alpha<\text { TOLER }) \text { THEN } \\
\boldsymbol{\Lambda}_{n+1 / 2}=\frac{1}{2}\left(\boldsymbol{\Lambda}_{n}+\boldsymbol{\Lambda}_{n+1}\right)
\end{gathered}
$$

ELSE

(iiia) Evaluate rotational vector and incremental rotational matrix

$$
\begin{aligned}
\Delta \mathbf{s}_{n+1 / 2} & =\frac{\mathbf{b}}{\|\mathbf{b}\|} \frac{\alpha}{2} \\
\Delta \mathbf{R}_{n+1 / 2} & =\Delta \mathbf{R}_{n+1 / 2}\left(\Delta \mathbf{s}_{n+1 / 2}\right)
\end{aligned}
$$

(iiib) Evaluate rotational matrix at $t_{n+1 / 2}$

$$
\boldsymbol{\Lambda}_{n+1 / 2}=\Delta \mathbf{R}_{n+1 / 2} \mathbf{\Lambda}_{n}
$$

$$
G_{\text {stat }}\left(\boldsymbol{\Phi}_{n}, \boldsymbol{\Phi}_{n+1}, \delta \boldsymbol{\Phi}\right)=\int_{\mathscr{M}_{0}} \mathbf{r} \cdot \delta \mathbf{E}_{n+1 / 2} \mathrm{~d} \mathscr{M}_{0}=\int_{\mathscr{M}_{0}} \mathbf{r} \cdot\left(\mathbf{B}_{n+1 / 2} \delta \boldsymbol{\Phi}\right) \mathrm{d} \mathscr{M}_{0}
$$

Matrices $\mathbf{B}_{n+1 / 2}, \mathbf{B}_{n+1}$ and vectors $\mathbf{p}_{n+1}, \pi_{n+1}$ and $\mathbf{r}$ are functions of configuration $\boldsymbol{\Phi}_{n+1}$. Note, that $\mathbf{r}$ is obtained from averaging the strains at time instants $t_{n}$ and $t_{n+1}$ (see (57), (59) and (60)).

\section{SPATIAL FINITE ELEMENT DISCRETIZATION}

In this section a finite element discretization, based on an isoparametric quadrilateral shell finite element, is presented. Approximation of the transverse shear field is performed by employing assumed natural strain (ANS) concept. $^{15}$

\subsection{Interpolation of shell geometry and physical fields}

The shell configuration in $\mathbb{R}^{3}$ can be completely described by the mid-surface position and the shell-director vector field (see Section 2). The adopted spatial finite element discretization relies on the isoparametric mapping from the parent element, which is represented as the biunit square with coordinates $\left(\xi^{1}, \xi^{2}\right) \in[-1,1] \times[-1,1] \subset \mathbb{R}^{2}$. In this section the subscripts $(\circ)_{n}$ or $(\circ)_{n+1}$ will be omitted.

In the standard manner the position vectors of a point on the mid-surface of a particular finite element $\Omega$ is given as

$$
\left.\mathbf{X}\left(\xi^{1}, \xi^{2}\right)\right|_{\Omega}=\sum_{I=1}^{4} N^{I}\left(\xi^{1}, \xi^{2}\right) \mathbf{X}_{I} \quad \text { and }\left.\quad \mathbf{x}\left(\xi^{1}, \xi^{2}\right)\right|_{\Omega}=\sum_{I=1}^{4} N^{I}\left(\xi^{1}, \xi^{2}\right) \mathbf{x}_{I}
$$

where $\mathbf{X}_{I}$ and $\mathbf{x}_{I}$ are vectors of nodal co-ordinates in the reference configuration (at time instant $t=0)$ and current configuration (e.g. at time instants $t_{n}$ or $\left.t_{n+1}\right) . N^{I}\left(\xi^{1}, \xi^{2}\right)$ are standard bilinear 
shape functions. In the following, the subscript $\left.\right|_{\Omega}$ will be omitted. It seems that for the four-node shell elements with five degrees-of-freedom per node the most appropriate way to evaluate reference nodal directors is by averaging nodal normals of the adjoined elements (e.g. References 16 and 18). Once the averaged normals are obtained for the reference finite element mesh, the interpolation of the shell-director field in reference and current configuration may be given in the standard way

$$
\mathbf{T}\left(\xi^{1}, \xi^{2}\right)=\sum_{I=1}^{4} N^{I}\left(\xi^{1}, \xi^{2}\right) \mathbf{T}_{I}, \quad \mathbf{t}\left(\xi^{1}, \xi^{2}\right)=\sum_{I=1}^{4} N^{I}\left(\xi^{1}, \xi^{2}\right) \mathbf{t}_{I}
$$

The isoparametric discretization of the shell leads to the following approximation of the displacement fields across the two-dimensional shell finite element

$$
\mathbf{U}\left(\xi^{1}, \xi^{2}, \xi\right)=\sum_{I=1}^{4} N^{I}\left(\xi^{1}, \xi^{2}\right) \mathbf{U}_{I}=\sum_{I=1}^{4} N^{I}\left(\xi^{1}, \xi^{2}\right)\left[\mathbf{u}_{I}+\xi\left(\mathbf{t}_{I}-\mathbf{T}_{I}\right)\right]
$$

Since $\mathbf{x}, \mathbf{t}, \mathbf{p}$ and $\pi$ are treated as mutually independent vector fields, the interpolation of surface and director momenta is given as

$$
\mathbf{p}\left(\xi^{1}, \xi^{2}\right)=\sum_{I=1}^{4} N^{I}\left(\xi^{1}, \xi^{2}\right) \mathbf{p}_{I}, \quad \pi\left(\xi^{1}, \xi^{2}\right)=\sum_{I=1}^{4} N^{I}\left(\xi^{1}, \xi^{2}\right) \pi_{I}
$$

The transverse shear field interpolation, based on ANS method, prescribes the transverse shear to vary linearly between two opposite element edges. In the middle of each edge the shear is calculated with respect to the edge direction

$$
\begin{aligned}
& 2 \bar{E}_{13}^{(0)}=\frac{1}{2}\left(1-\xi^{2}\right) 2 E_{13}^{(0) A}+\frac{1}{2}\left(1+\xi^{2}\right) 2 E_{13}^{(0) C} \\
& 2 \bar{E}_{23}^{(0)}=\frac{1}{2}\left(1-\xi^{1}\right) 2 E_{23}^{(0) D}+\frac{1}{2}\left(1+\xi^{1}\right) 2 E_{23}^{(0) B}
\end{aligned}
$$

where the mid-surface points $\mathbf{x}^{L}, L=A, B, C, D$ are defined with $\mathbf{x}^{L}=\frac{1}{2} \mathbf{x}\left(\check{\xi}_{M}^{1}, \xi_{M}^{2}\right)+\frac{1}{2} \mathbf{x}\left(\xi_{N}^{1}, \xi_{N}^{2}\right)$ and $(L, M, N) \in\{(A, 1,2) ;(B, 2,3) ;(C, 3,4) ;(D, 1,4)\}$.

Spatial discretization of the weak form of the equations of motion discretized in time requires interpolation of test functions, derivatives of the mid-surface displacement and rotation fields and derivatives of test functions. In accordance with Section 4.1 they may be expressed as

$$
\begin{aligned}
& \delta \mathbf{t}=\sum_{I=1}^{4} N^{I} \delta \mathbf{t}_{I}, \quad \mathbf{u}_{, \alpha}=\sum_{I=1}^{4} N_{, \alpha}^{I} \mathbf{u}_{I}, \quad \mathbf{t}_{, \alpha}=\sum_{I=1}^{4} N_{, \alpha}^{I} \mathbf{t}_{I} \\
& \delta \mathbf{u}_{, \alpha}=\sum_{I=1}^{4} N_{, \alpha}^{I} \delta \mathbf{u}_{I}, \quad \delta \mathbf{t}_{, \alpha}=\sum_{I=1}^{4} N_{, \alpha}^{I} \delta \mathbf{t}_{I}
\end{aligned}
$$

Nodal vector $\mathbf{t}_{I}$ is obtained from (10) and $\delta \mathbf{t}_{l}$ is given by (56). Thus, it is necessary to construct a local orthonormal basis $\left\{\mathbf{E}_{i}\right\}$ at each nodal point (see Section 2.1.2).

Linearisation process further demands the second directional derivatives $\Delta(\delta \mathbf{t})$ and $\Delta\left(\delta \mathbf{t}_{, \alpha}\right)$, which are interpolated as

$$
\Delta(\delta \mathbf{t})=\sum_{l=1}^{4} N^{I} \Delta\left(\delta \mathbf{t}_{l}\right), \quad \Delta\left(\delta \mathbf{t}_{, \alpha}\right)=\sum_{I=1}^{4} N_{, \alpha}^{I} \Delta\left(\delta \mathbf{t}_{l}\right)
$$

Explicit expression for $\Delta\left(\delta \mathbf{t}_{l}\right)$ is provided in Section 5.1. 


\subsection{Discrete weak form of the equations of motion}

By inserting the above interpolations into the time-discretized weak form of the equations of motion the fully discrete version of (44) is obtained. The inertial and static parts are defined as

$$
\begin{aligned}
G_{\text {ine }}\left(\boldsymbol{\Phi}_{n}, \boldsymbol{\Phi}_{n+1}, \delta \boldsymbol{\Phi}\right) & =\bigcup_{e=1}^{N_{\text {elem }}} \frac{1}{\Delta t} \int_{A_{e}} \delta \boldsymbol{\Phi}^{\mathrm{T}}\left[\begin{array}{cc}
\mathbf{p}_{n+1}-\mathbf{p}_{n} & \mathbf{0} \\
\mathbf{0} & \pi_{n+1}-\pi_{n}
\end{array}\right] \mathrm{d} A_{e} \\
G_{\text {stat }}\left(\boldsymbol{\Phi}_{n}, \boldsymbol{\Phi}_{n+1}, \delta \boldsymbol{\Phi}\right) & =\bigcup_{e=1}^{N_{\text {elem }}} \int_{A_{e}} \mathbf{r} \cdot\left(\mathbf{B}_{n+1 / 2} \delta \boldsymbol{\Phi}\right) \mathrm{d} A_{e}
\end{aligned}
$$

Remark 4.1. The integrals over the mid-surface of the quadrilateral finite element are evaluated by $2 \times 2$ quadrature. At each integration point a product of vectors and $\backslash$ or matrices is required. It is therefore no need to use curvilinear convective co-ordinate system, as long as all quantities in one integration point are related to the same co-ordinate system. It is thus suitable to introduce a Cartesian co-ordinate system at each mid-surface integration point, since the expressions in such system take simpler form. They may also provide a basis for future inelastic material description within the present dynamic shell formulation. ${ }^{19}$ Details, regarding the implementation of such systems may be found in Reference 20 .

Remark 4.2. The conservation laws of the total linear and angular momentum follow from the fully discrete weak form of the equations of motion only if interpolations (71), (74) and (75) are used for the rotational part of the formulation. For details see Reference 2.

\section{LINEARIZATION ASPECTS}

The discrete weak form of the equations of motion for the present dynamic shell formulation represents a set of non-linear equations with kinematic variables (mid-surface nodal displacements and nodal rotational parameters) at time instant $t_{n+1}$ as the only unknowns. The solution of these equations is obtained by using the Newton-Raphson solution procedure. An essential ingredient of this procedure is the linearization of the discrete weak form which yields a tangent stiffness operator. In this section the basic steps of the process are described.

\subsection{Derivatives of test functions}

In the linearization process, the directional derivatives of test functions, $\delta \boldsymbol{\Phi}=[\delta \mathbf{u}, \delta \mathbf{t}]^{\mathrm{T}}$, in the direction of $\Delta \boldsymbol{\Phi}_{n+1}$ are needed. Formally, they may be obtained as

$$
D(\delta \boldsymbol{\Phi}) \cdot \Delta \boldsymbol{\Phi}_{n+1}=\left.\frac{\mathrm{d}}{\mathrm{d} \varepsilon}\right|_{\varepsilon=0} \delta \boldsymbol{\Phi}\left(\boldsymbol{\Phi}_{n}+\varepsilon \Delta \boldsymbol{\Phi}_{n+1}\right)=\left.\frac{\mathrm{d}}{\mathrm{d} \varepsilon}\right|_{\varepsilon=0} \delta \boldsymbol{\Phi}_{\varepsilon}
$$

It can be easily verified that $D(\delta \mathbf{u}) \cdot \Delta \boldsymbol{\Phi}_{n+1}$ is zero, which is in contrast with $D(\delta \mathbf{t}) \cdot \Delta \boldsymbol{\Phi}_{n+1}$, also denoted as $\Delta(\delta \mathbf{t})$. The later derivative may be obtained by constructing a one-parameter curve $(\delta \mathbf{t})_{\varepsilon}$, and by performing its differentiation with respect to $\varepsilon$ at $\varepsilon=0$.

We remind that the test function $\delta \mathbf{t}$ needs to satisfy the condition $\delta \mathbf{t} \cdot \mathbf{t}_{n+1 / 2}=0$, which is an algorithmic approximation of constraint (36). Accordingly, a one-parameter curve, $(\delta \mathrm{t})_{\varepsilon}$, may be constructed as $(\delta \mathbf{t})_{\varepsilon}=\delta \mathbf{s}_{L} \times\left(\tilde{\mathbf{t}}_{n+1 / 2}\right)_{\varepsilon}\left(\right.$ see (38)) where, $\tilde{\mathbf{t}}_{n+1 / 2}$ is a unit vector $\tilde{\mathbf{t}}_{n+1 / 2}=\mathbf{t}_{n+1 / 2} /\left\|\mathbf{t}_{n+1 / 2}\right\|$. 
Having constructed $(\delta \mathbf{t})_{\varepsilon}$, the expression for $\Delta(\delta \mathbf{t})$ may be obtained after some straightforward manipulations. By multiplying the result with an arbitrary vector $\mathbf{b} \in \mathbb{R}^{3}$, we obtain

$$
\mathbf{b} \cdot \Delta(\delta \mathbf{t})=\delta \hat{\mathbf{T}}^{\mathrm{T}}\left(\frac{\mathbf{b} \cdot \tilde{\mathbf{t}}_{n+1 / 2}}{\left\|\mathbf{t}_{n}+\mathbf{t}_{n+1}\right\|}\right)\left[\overline{\boldsymbol{\Lambda}}_{n+1 / 2}^{\mathrm{T}}\left(\tilde{\mathbf{t}}_{n+1 / 2} \otimes \tilde{\mathbf{t}}_{n+1 / 2}-\mathbf{I}\right) \overline{\boldsymbol{\Lambda}}_{n+1}\right] \Delta \hat{\mathbf{T}}_{n+1}
$$

where $\delta \mathbf{t}=\overline{\mathbf{\Lambda}}_{n+1 / 2} \delta \hat{\mathbf{T}}$ and $\Delta \mathbf{t}_{n+1}=\overline{\mathbf{\Lambda}}_{n+1} \Delta \hat{\mathbf{T}}_{n+1}$.

\subsection{Newton-Raphson method}

The present problem may be stated as: Assuming that configuration and momenta (velocities) at time instant $t_{n}$ are known, find configuration at time instant $t_{n+1}$, such that the discretized functional $G_{\mathrm{dyn}}\left(\mathbf{Z}_{n}, \boldsymbol{\Phi}_{n+1}, \delta \Phi\right)=0$ for any test functions $\delta \boldsymbol{\Phi}$ related to intermediate configuration. The solution of the above non-linear problem is pursued by employing the Newton-Raphson method. Therefore, a linearization of $G_{\mathrm{dyn}}\left(\mathbf{Z}_{n}, \boldsymbol{\Phi}_{n+1}, \delta \boldsymbol{\Phi}\right)$ is required. The Newton-Raphson procedure at the configuration $\boldsymbol{\Phi}_{n+1}=\widehat{\boldsymbol{\Phi}}_{n+1}$ may be expressed in the form

$$
D G_{\mathrm{dyn}}\left(\mathbf{Z}_{n}, \widehat{\boldsymbol{\Phi}}_{n+1}^{(i)}, \delta \boldsymbol{\Phi}\right) \cdot \Delta \boldsymbol{\Phi}_{n+1}^{(i)}=-G_{\mathrm{dyn}}^{(i)}
$$

where the left-hand-side supplies the tangent stiffness operator. By assuming dead loading, the tangent stiffness operator may be conventionally split into inertial (dynamic), material and geometric part

$$
\begin{aligned}
& D G_{\mathrm{dyn}}\left(\mathbf{Z}_{n}, \boldsymbol{\Phi}_{n+1}, \delta \boldsymbol{\Phi}\right) \cdot \Delta \boldsymbol{\Phi}_{n+1}=D_{D} G_{\mathrm{dyn}}\left(\mathbf{Z}_{n}, \boldsymbol{\Phi}_{n+1}, \delta \boldsymbol{\Phi}\right) \cdot \Delta \boldsymbol{\Phi}_{n+1} \\
& \quad+D_{M} G_{\mathrm{dyn}}\left(\boldsymbol{\Phi}_{n}, \boldsymbol{\Phi}_{n+1}, \delta \boldsymbol{\Phi}\right) \cdot \Delta \boldsymbol{\Phi}_{n+1}+D_{G} G_{\mathrm{dyn}}\left(\boldsymbol{\Phi}_{n}, \boldsymbol{\Phi}_{n+1}, \delta \boldsymbol{\Phi}\right) \cdot \Delta \boldsymbol{\Phi}_{n+1}
\end{aligned}
$$

\subsection{Dynamics predictors and updates of kinematic variables}

To start the Newton-Raphson iteration, the configuration and velocities at time $t_{n+1}$ at first iteration $i=0$ have to be predicted. Various possibilities exist for dynamic predictors. Either choice does not effect the final result but may effect the convergence characteristics:

(i) Constant deformation is assumed between $t_{n}$ and $t_{n+1}$ at iteration $i=0$. This leads to $\boldsymbol{\Phi}_{n+1}=$ $\Phi_{n}$ and reversal of momenta (velocities) in the first iteration $\Pi_{n+1}=-\Pi_{n}$ (see (50)).

(ii) Constant velocity is assumed between $t_{n}$ and $t_{n+1}$ at iteration $i=0$. This leads to $\boldsymbol{\Pi}_{n+1}=\boldsymbol{\Pi}_{n}$, while the configuration at iteration $i=0$ is defined as $\mathbf{x}_{n+1}=\mathbf{x}_{n}+\mathbf{p}_{n} / A_{p_{0}} \Delta t, \mathbf{t}_{n+1}=\mathbf{t}_{n}+$ $\pi / I_{\rho_{0}} \Delta t$. Numerical examples in Section 6 are computed using this scheme.

The updates of kinematic variables take place at the nodal level. Special consideration must be given to the implementation of the rotational updates. The procedure, employed in this work, is given in Box 2. The incremental nodal rotation vector at iteration $i$ is obtained as

$$
\left(\Delta \mathbf{s}_{l}\right)_{n+1}^{i}=\left(\mathbf{t}_{l}\right)_{n+1}^{i} \times\left(\Delta \mathbf{t}_{l}\right)_{n+1}^{i}=\left(\boldsymbol{\Lambda}_{l}\right)_{n+1}^{i}\left[\overline{\mathbf{T}}_{I} \times\left(\Delta \overline{\mathbf{T}}_{l}\right)_{n+1}^{i}\right]
$$

Since $\overline{\mathbf{T}}_{I}=[0,0,1]^{\mathrm{T}}$ and transpose of the vector $\left(\Delta \overline{\mathbf{T}}_{I}\right)_{n+1}^{i}$ is $\left[\Delta \bar{T}_{I}^{1}, \Delta \bar{T}_{I}^{2}, 0\right]_{n+1}^{i}$, the cross product in brackets may be explicitly evaluated (see Box 2 ).

Remark 5.1. It is also possible to assume constant acceleration within the time-step for the dynamic predictor. This leads to $\ddot{\boldsymbol{\Phi}}_{n+1 / 2}=\ddot{\boldsymbol{\Phi}}_{n+1}=\ddot{\boldsymbol{\Phi}}_{n}$ and definition of velocity and configuration 
Box 2. Update of nodal rotational variables algorithm

(i) Evaluate nodal rotational matrix $\Lambda_{I}^{0}$

$$
\Lambda_{I}^{0}=\left[\mathbf{E}_{1}, \mathbf{E}_{2}, \mathbf{T}\right]_{I}
$$

(ii) Search for the solution at the end of the time interval $\left[t_{n}, t_{n+1}\right]$

(iii) Solve the non-linear set of equations by N-R procedure and get nodal rotational parameters

$$
D G_{\mathrm{dyn}}^{(i)} \cdot \Delta \boldsymbol{\Phi}_{n+1}^{(i)}=-G_{\mathrm{dyn}}^{(i)} \Rightarrow\left(\Delta \hat{\mathbf{T}}_{I}\right)_{n+1}^{i}=\left\{\begin{array}{l}
\Delta \bar{T}^{1} \\
\Delta \bar{T}^{2}
\end{array}\right\}_{n+1}^{i}
$$

(iv) Update nodal rotational matrix and nodal shell director

(iva) Evaluate incremental rotational vector

$$
\left(\Delta \mathbf{s}_{I}\right)_{n+1}^{i+1}=\left\{\begin{array}{l}
\left(\Lambda_{12}\right)_{I} \\
\left(\Lambda_{22}\right)_{I} \\
\left(\Lambda_{32}\right)_{I}
\end{array}\right\}_{n+1}^{i}\left(\Delta \bar{T}^{1}\right)_{n+1}^{i}-\left\{\begin{array}{l}
\left(\Lambda_{11}\right)_{I} \\
\left(\Lambda_{21}\right)_{I} \\
\left(\Lambda_{31}\right)_{I}
\end{array}\right\}_{n+1}^{i}\left(\Delta \bar{T}^{2}\right)_{n+1}^{i}
$$

(ivb) Evaluate incremental rotational matrix

$$
\left(\Delta \mathbf{R}_{I}\right)_{n+1}^{i+1}=\mathbf{I}+\frac{\sin \left\|\left(\Delta \mathbf{s}_{I}\right)_{n+1}^{i+1}\right\|}{\left\|\left(\Delta \mathbf{s}_{I}\right)_{n+1}^{i+1}\right\|}\left(\Delta \mathbf{S}_{I}\right)_{n+1}^{i+1}+\frac{1-\cos \left\|\left(\Delta \mathbf{s}_{I}\right)_{n+1}^{i+1}\right\|}{\left\|\left(\Delta \mathbf{s}_{I}\right)_{n+1}^{i+1}\right\|^{2}}\left[\left(\Delta \mathbf{S}_{I}\right)_{n+1}^{i+1}\right]^{2}
$$

(ivc) Update rotational matrix

IF $t_{n}=t_{0}$ AND $i=0$ THEN $\left(\boldsymbol{\Lambda}_{I}\right)_{n+1}^{i+1}=\left(\Delta \mathbf{R}_{I}\right)_{n+1}^{i+1} \Lambda_{I}^{0}$

$\operatorname{ELSE}\left(\boldsymbol{\Lambda}_{I}\right)_{n+1}^{i+1}=\left(\Delta \mathbf{R}_{I}\right)_{n+1}^{i+1}\left(\boldsymbol{\Lambda}_{I}\right)_{n+1}^{i}$

(ivd) Update shell director vector

(v) Check for convergence

$$
\left(\mathbf{t}_{I}\right)_{n+1}^{i+1}=\left\{\begin{array}{l}
\left(\Lambda_{13}\right)_{I} \\
\left(\Lambda_{23}\right)_{I} \\
\left(\Lambda_{33}\right)_{I}
\end{array}\right\}_{n+1}^{i}
$$

$$
\begin{aligned}
& \text { IF }\left\|\mathbf{G}^{(i+1)}\right\|>\text { TOLER THEN } i=i+1 \text { and GOTO (iii) } \\
& \text { ELSE } t_{n}=t_{n}+\Delta t, t_{n+1}=t_{n+1}+\Delta t, i=0 \text { and GOTO (ii) }
\end{aligned}
$$

at $t_{n+1}$ as $\dot{\boldsymbol{\Phi}}_{n+1}=\ddot{\boldsymbol{\Phi}}_{n} \Delta t+\dot{\boldsymbol{\Phi}}_{n}$ and $\boldsymbol{\Phi}_{n+1}=\left(\Delta t^{2} / 2\right) \ddot{\boldsymbol{\Phi}}_{n}+\Delta t \dot{\boldsymbol{\Phi}}_{n}+\boldsymbol{\Phi}_{n}$, respectively. However, with this choice, some modifications of the present formulation are needed.

Remark 5.2. The updates of rotational parameters may be also performed in terms of quaternion parameters as presented in References 7 and 2. In this way the orthogonality of nodal rotational matrix may be better controlled during the computations.

\subsection{Dynamic part of the tangent stiffness operator}

The dynamic part of the fully discrete weak form of the equations of motion is functional of unknown configuration $\boldsymbol{\Phi}_{n+1}$ only. Linearization of (53) renders an inertial (dynamic) part of the 
stiffness operator. It may be written as

$$
\begin{aligned}
& D_{D} G_{\text {dyn }}\left(\boldsymbol{\Phi}_{n}, \boldsymbol{\Pi}_{n}, \boldsymbol{\Phi}_{n+1}, \delta \boldsymbol{\Phi}\right) \cdot \Delta \boldsymbol{\Phi}_{n+1} \\
& \quad=\bigcup_{e=1}^{N_{\text {elem }}} \int_{A_{e}}\left[\frac{2 A_{\rho_{0}}}{(\Delta t)^{2}} \Delta \mathbf{u}_{n+1} \cdot \delta \mathbf{u}+\frac{2 I_{\rho_{0}}}{(\Delta t)^{2}} \Delta \mathbf{t}_{n+1} \cdot \delta \mathbf{t}+\frac{1}{\Delta t}\left(\pi_{n+1}-\pi_{n}\right) \cdot \Delta(\delta \mathbf{t})\right] \mathrm{d} A_{e}
\end{aligned}
$$

The third part of (83) may be evaluated by using (79).

\subsection{Material part of the tangent stiffness operator}

The material part of the stiffness operator arises from the linearization of constitutive equations. Since constitutive matrix $\mathbf{C}$ and strain measures $\mathbf{E}_{n}$ do not depend on $\boldsymbol{\Phi}_{n+1}$, the following expression may be obtained when performing the linearisation of the algorithmic stress resultants vector $\mathbf{r}$

$$
D \mathbf{r} \cdot \Delta \boldsymbol{\Phi}_{n+1}=C_{\frac{1}{2}} \mathbf{B}_{n+1} \Delta \Phi_{n+1}
$$

The definitions of algorithmic stress resultant vector, $\mathbf{r}$, strain-displacement operator, $\mathbf{B}_{n+1}$, and constitutive matrix $\mathbf{C}$ are given in Section 3.4. Note that factor $\frac{1}{2}$ stems from the definition of $\mathbf{r}$ (see (60) and (63)). By using (84) the material part of the stiffness operator may be written as

$$
D_{M} G_{\text {dyn }} \cdot \Delta \boldsymbol{\Phi}_{n+1}=\bigcup_{e=1}^{N_{\text {elem }}} \int_{A_{e}}\left(\mathbf{B}_{n+1 / 2} \delta \Phi\right) \cdot\left(C \frac{1}{2} \mathbf{B}_{n+1} \Delta \boldsymbol{\Phi}_{n+1}\right) \mathrm{d} A_{e}
$$

where $\mathbf{B}_{n+1 / 2}=\frac{1}{2}\left(\mathbf{B}_{n}+\mathbf{B}_{n+1}\right)$. It may be noted from (84) and (85) that the material part of the finite element stiffness matrix is non-symmetric.

\subsection{Geometric part of the tangent stiffness operator}

The geometric part of the stiffness operator arises from the linearization of the geometric part of $G_{\text {stat }}\left(\boldsymbol{\Phi}_{n}, \boldsymbol{\Phi}_{n+1}, \delta \boldsymbol{\Phi}\right)$, i.e. from the linearisation of $\delta \mathbf{E}$ (see (67)). It may be written in a compact form as

$$
\begin{aligned}
D_{G} G_{\mathrm{dyn}} \cdot \Delta \boldsymbol{\Phi}_{n+1} & =\bigcup_{e=1}^{N_{\text {clem }}} \int_{A_{e}} \mathbf{r} \cdot\left[D(\delta \mathbf{E}) \cdot \Delta \boldsymbol{\Phi}_{n+1}\right] \mathrm{d} A_{e} \\
& =\bigcup_{e=1}^{N_{\text {clem }}} \int_{A_{e}} \mathbf{r} \cdot\left\{\frac{1}{2}\left[D\left(\mathbf{B}_{n+1}\right) \cdot \Delta \boldsymbol{\Phi}_{n+1}\right] \delta \boldsymbol{\Phi}+\mathbf{B}_{n+1 / 2} D(\delta \boldsymbol{\Phi}) \cdot \Delta \boldsymbol{\Phi}_{n+1}\right\} \mathrm{d} A_{e}
\end{aligned}
$$

where $D\left(\mathbf{B}_{n+1}\right) \cdot \Delta \boldsymbol{\Phi}_{n+1}$ is the directional derivative of strain-displacement operator $\mathbf{B}_{n+1}$ in the direction of $\Delta \boldsymbol{\Phi}_{n+1}$. Results of the linearization $D(\delta \mathbf{E}) \cdot \Delta \boldsymbol{\Phi}_{n+1}$ may be presented as

$$
\begin{aligned}
& D\left(\delta E_{\alpha \beta}^{(0)}\right) \cdot \Delta \boldsymbol{\Phi}_{n+1}=\frac{1}{2}\left[\frac{1}{2}\left(\Delta \mathbf{u}_{n+1, \alpha} \cdot \delta \mathbf{u},{ }_{\beta}+\Delta \mathbf{u}_{n+1, \beta} \cdot \delta \mathbf{u}, \alpha\right)\right] \\
& D\left(2 \delta E_{\alpha 3}^{(0)}\right) \cdot \Delta \boldsymbol{\Phi}_{n+1}=\frac{1}{2}\left(\Delta \mathbf{u}_{n+1}, \alpha \cdot \delta \mathbf{t}+\delta \mathbf{u}, \alpha \cdot \Delta \mathbf{t}_{n+1}\right)+\underline{\frac{1}{2}\left(\mathbf{x}_{n, \alpha}+\mathbf{x}_{n+1, \alpha}\right) \cdot \Delta(\delta \mathbf{t}),} \\
& D\left(\delta E_{\alpha \beta}^{(1)}\right) \cdot \Delta \boldsymbol{\Phi}_{n+1}=\frac{1}{2}\left[\frac{1}{2}\left(\Delta \mathbf{u}_{n+1, \alpha} \cdot \delta \mathbf{t}, \beta+\Delta \mathbf{u}_{n+1, \beta} \cdot \delta \mathbf{t}, \alpha\right)\right]
\end{aligned}
$$




$$
\begin{aligned}
& +\frac{1}{2}\left[\frac{1}{2}\left(\delta \mathbf{u}, \alpha \cdot \Delta \mathbf{t}_{n+1}, \beta+\delta \mathbf{u}, \beta \cdot \Delta \mathbf{t}_{n+1}, \alpha\right)\right]+\underline{\frac{1}{2}\left[\frac{1}{2}\left(\mathbf{x}_{n, \alpha}+\mathbf{x}_{n+1}, \alpha\right) \cdot \Delta(\delta \mathbf{t}, \beta)\right]} \\
& +\underline{\frac{1}{2}\left[\frac{1}{2}\left(\mathbf{x}_{n}, \beta+\mathbf{x}_{n+1}, \beta\right) \cdot \Delta(\delta \mathbf{t}, \alpha)\right]}
\end{aligned}
$$

The underlined terms relate to the second part of $(86)_{2}$, and the other terms relate to the first part of $(86)_{2}$. By using (86), (87) and spatial interpolations defined in Section 4 , the components of the geometric finite element stiffness matrix may be recovered from the following relations

$$
\begin{aligned}
n^{\alpha \beta} D\left(\delta E_{\alpha \beta}^{(0)}\right) \cdot \Delta \boldsymbol{\Phi}_{n+1}= & \sum_{I=1}^{4} \sum_{K=1}^{4} \delta \mathbf{u}_{I} \cdot\left(\Delta \mathbf{u}_{K}\right)_{n+1} \frac{1}{2}\left(\frac{1}{2} n^{\alpha \beta}\left(N_{, \alpha}^{K} N_{, \beta}^{I}+N_{, \beta}^{K} N_{, \alpha}^{I}\right)\right) \\
m^{\alpha \beta} D\left(\delta E_{\alpha \beta}^{(1)}\right) \cdot \Delta \boldsymbol{\Phi}_{n+1}= & \sum_{I=1}^{4} \sum_{K=1}^{4} \delta \mathbf{t}_{I} \cdot\left(\Delta \mathbf{u}_{K}\right)_{n+1} \frac{1}{2}\left(\frac{1}{2} m^{\alpha \beta}\left(N_{, \alpha}^{K} N_{, \beta}^{I}+N_{, \beta}^{K} N_{, \alpha}^{I}\right)\right) \\
& +\sum_{I=1}^{4} \sum_{K=1}^{4} \delta \mathbf{u}_{I} \cdot\left(\Delta \mathbf{t}_{K}\right)_{n+1} \frac{1}{2}\left(\frac{1}{2} m^{\alpha \beta}\left(N_{, \alpha}^{I} N_{, \beta}^{K}+N_{, \beta}^{I} N_{, \alpha}^{K}\right)\right) \\
& +\sum_{I=1}^{4} \Delta\left(\delta \mathbf{t}_{I}\right) \cdot\left[\frac{1}{2} m^{\alpha \beta}\left(\mathbf{x}_{n+1 / 2, \alpha} N_{, \beta}^{I}+\mathbf{x}_{n+1 / 2, \beta} N_{, \alpha}^{I}\right)\right] \\
q^{\alpha 3} D\left(2 \delta E_{\alpha 3}^{(0)}\right) \cdot \Delta \boldsymbol{\Phi}_{n+1}= & \sum_{I=1}^{4} \sum_{K=1}^{4} \delta \mathbf{t}_{I} \cdot\left(\Delta \mathbf{u}_{K}\right)_{n+1}\left(q^{\alpha 3} N_{, \alpha}^{K} N^{I}\right) \\
& +\sum_{I=1}^{4} \sum_{K=1}^{4} \delta \mathbf{u}_{I} \cdot\left(\Delta \mathbf{t}_{K}\right)_{n+1}\left(q^{\alpha 3} N_{, \alpha}^{I} N^{K}\right) \\
& +\sum_{I=1}^{4} \Delta\left(\delta \mathbf{t}_{I}\right) \cdot\left(q^{\alpha 3} \mathbf{x}_{n+1 / 2, \alpha} N^{I}\right)
\end{aligned}
$$

\section{NUMERICAL EXAMPLES}

In this section a set of numerical examples is presented in order to illustrate the performance capabilities of the described dynamic shell model and associated numerical formulation. In all examples, the non-linear equations are solved by the full Newton-Raphson method. The global convergence is achieved when the Euclidean norm of the residual forces is less than $1.0 \times 10^{-6}$.

Example 6.1: Motion of a short cylinder under impulsive line loads. With this example, the dynamics of a short elastic cylinder under impulsive loading, Simo and Tarnow ${ }^{3}$ studied performance of different numerical time-integration schemes, i.e. energy-momentum method, mid-point rule and trapezoidal rule. The geometry characteristics, the finite element mesh, the material parameters and the loading conditions are given in Figure 1. At the reference position the initial conditions are prescribed to be zero. The shell is subjected to a short impulsive external loads from 0 till $1 \mathrm{~s}$. During the computational process the time interval $[0,25] \mathrm{s}$ is divided into 1250 time subintervals with $\Delta t=0.02 \mathrm{~s}$. When the loads are removed, the structure exhibits a complex motion, which is evident from Figure 2, where the deformed shapes of a cylinder are depicted for every $2 \mathrm{~s}$ and shown from two different perspectives. Figures 3 and 4 illustrate that three vector components of the total linear momentum, as well as three vector components of the total angular momentum are preserved constant during the free motion by the adopted time-integration algorithm. This feature may be observed even for mid-point rule, while trapezoidal rule does not exactly conserve angular momentum as shown in Reference 3. As expected, the energy-momentum method preserves the 


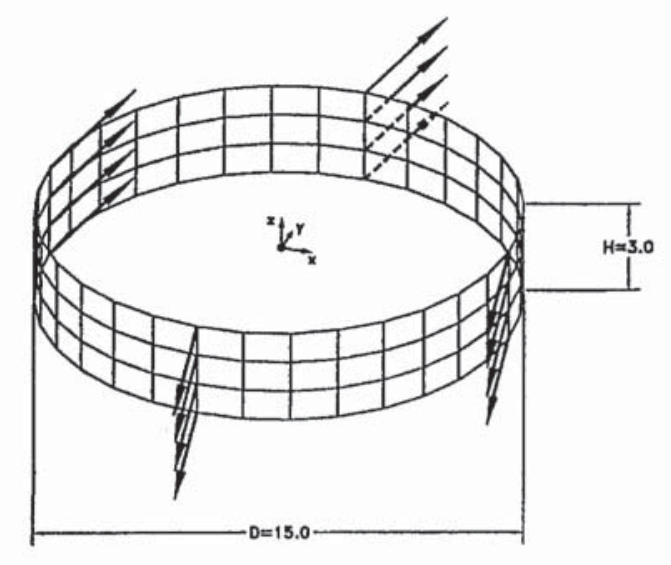

$\begin{array}{ll}\text { R: } & 7.5 \\ \text { Height: } & 3.0 \\ \text { Thickness: } & 0.02 \\ \text { Modulus E: } & 2 \cdot 10^{8} \\ \text { Modulus v: } & 0.5 \\ \text { Density: } & 1.0 \\ \text { Rot. inertia: } & 6.66 \cdot 10^{-7}\end{array}$

\begin{tabular}{|l|l|l|l|l|}
\hline Angle $\alpha$ & 0 & $\pi / 2$ & $\pi$ & $3 \pi / 2$ \\
\hline Nodal loads & {$[0,-1,-1]^{T} p(t)$} & {$[1,1,1]^{T} p(t)$} & {$[1,1,1]^{T} p(t)$} & {$[0,-1,-1]^{T} p(t)$} \\
\hline
\end{tabular}

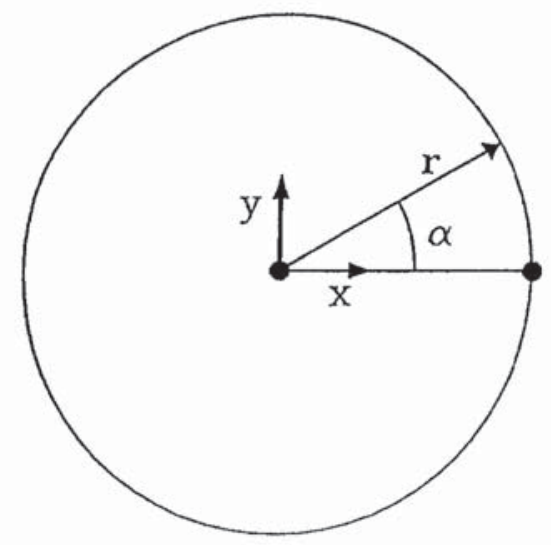

\begin{tabular}{|l|l|}
\hline Time & $p(t)$ \\
\hline 0.0 & 0.0 \\
\hline 0.5 & 5.0 \\
\hline 1.0 & 0.0 \\
\hline
\end{tabular}

Figure 1. Short cylinder: geometry, material characteristics and loading conditions

total input energy for the undamped system (Figure 5). In Figure 5 the periodic oscillations of internal and kinetic energy may be observed. It is interesting to note that the stress-free configurations arise approximately every second. The computed total energy is slightly higher of the value presented in Reference 3. This difference is a consequence of different constants taken for the rotational inertia $I_{\rho_{0}}\left(\rho_{0} H^{3} / 12=6.66 \times 10^{-7}\right.$ in the present case and $3.33 \times 10^{-5}$ in Reference 3$)$. In Figure 6 the convergence characteristic for various time step numbers $(400,600,800$ and 1000$)$ are depicted. The Euclidean norms of the residuals $\|\mathbf{G}\|$ clearly illustrate the quadratic rate of asymptotic convergence typical for the Newton-Raphson based iterative procedure.

Example 6.2: Dynamic snap-through of a spherical cap under impulsive ring loading. Geometry characteristics, material parameters, finite element mesh and loading conditions for this example are given in Figure 7. The radius of the sphere is $R=10$, the radius of the hole is $R=3 \cdot 88$, 

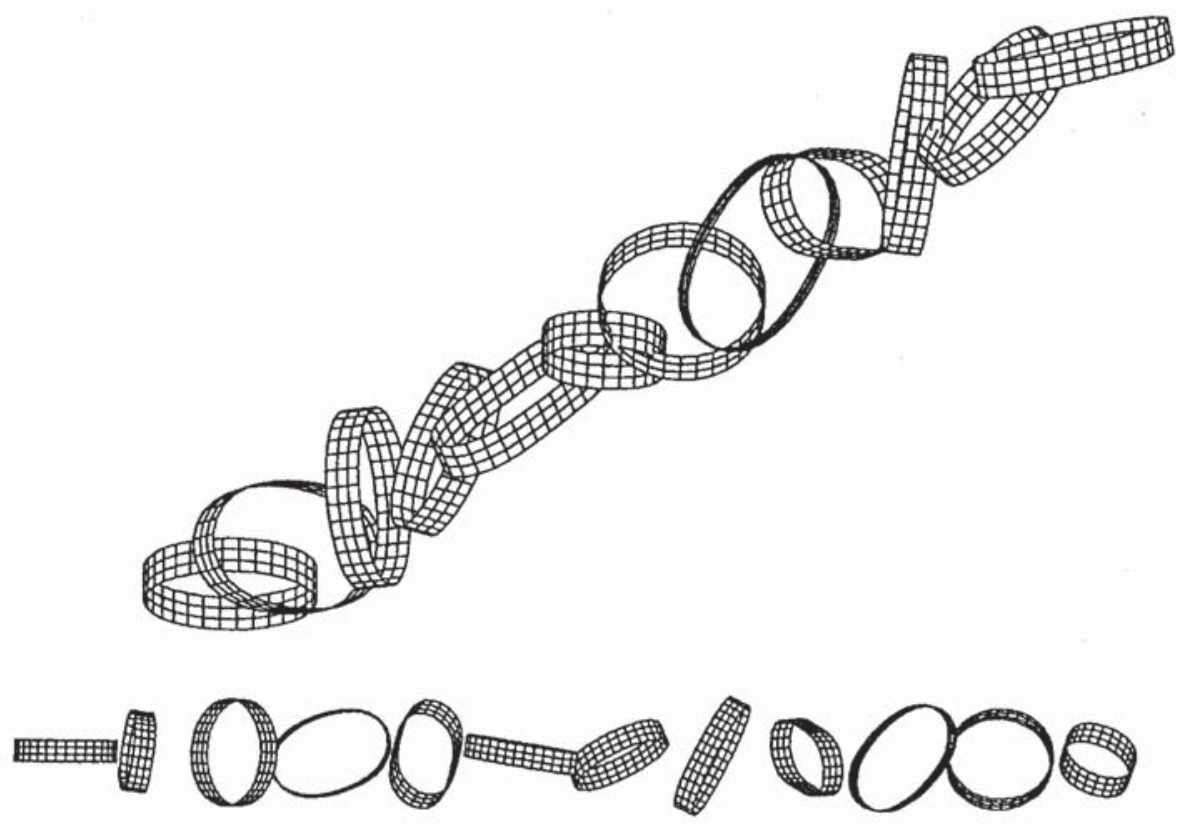

Figure 2. Short cylinder: sequence of deformed finite element meshes in the time interval $[0,25] \mathrm{s}$, depicted for every $2 \mathrm{~s}$ and shown from two different perspectives

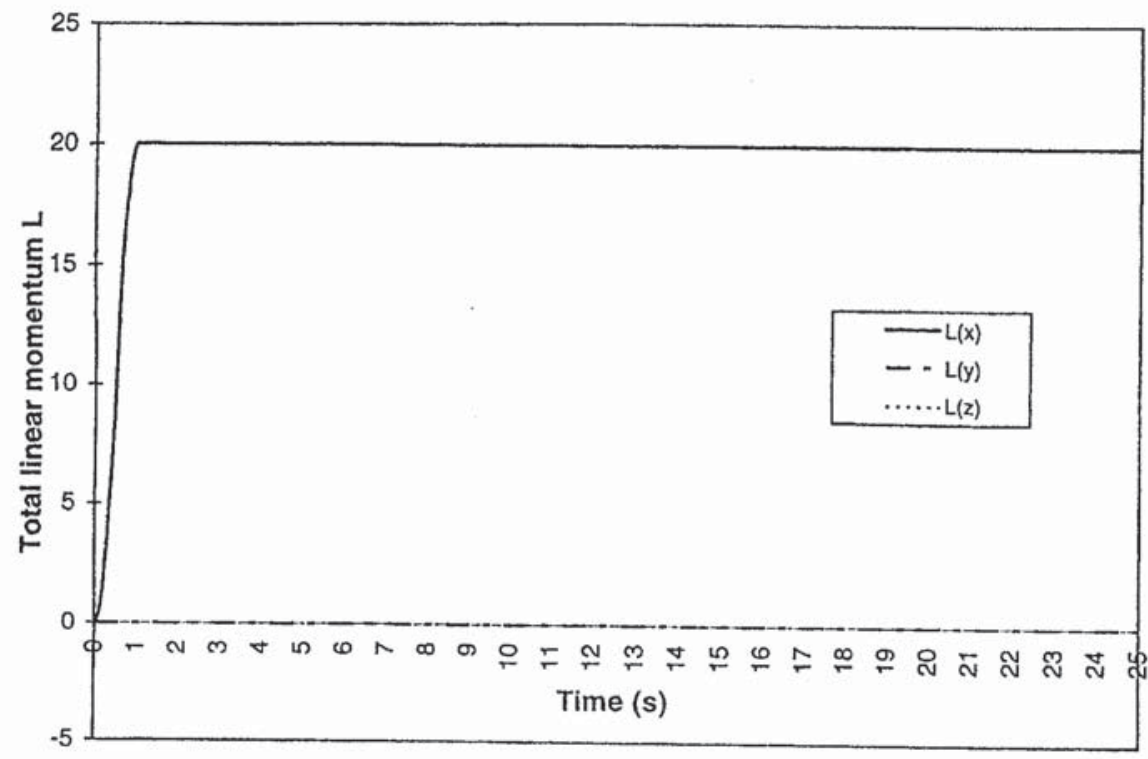

Figure 3. Short cylinder: time history of total linear momentum 


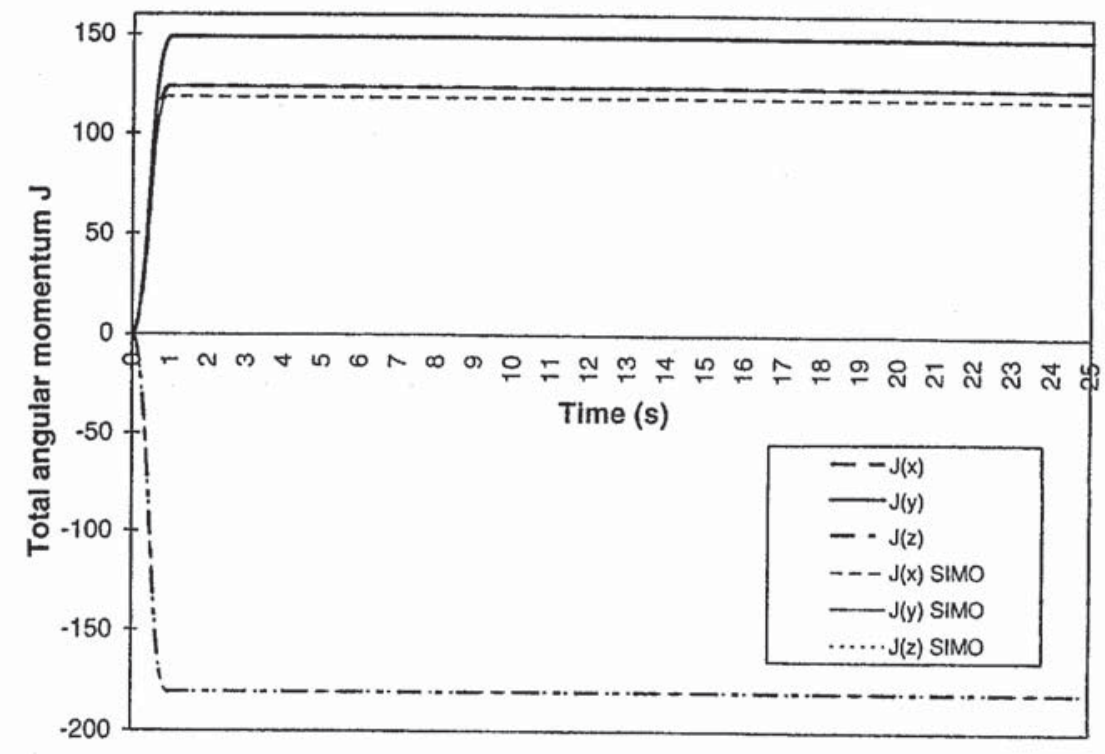

Figure 4. Short cylinder: time history of total angular momentum

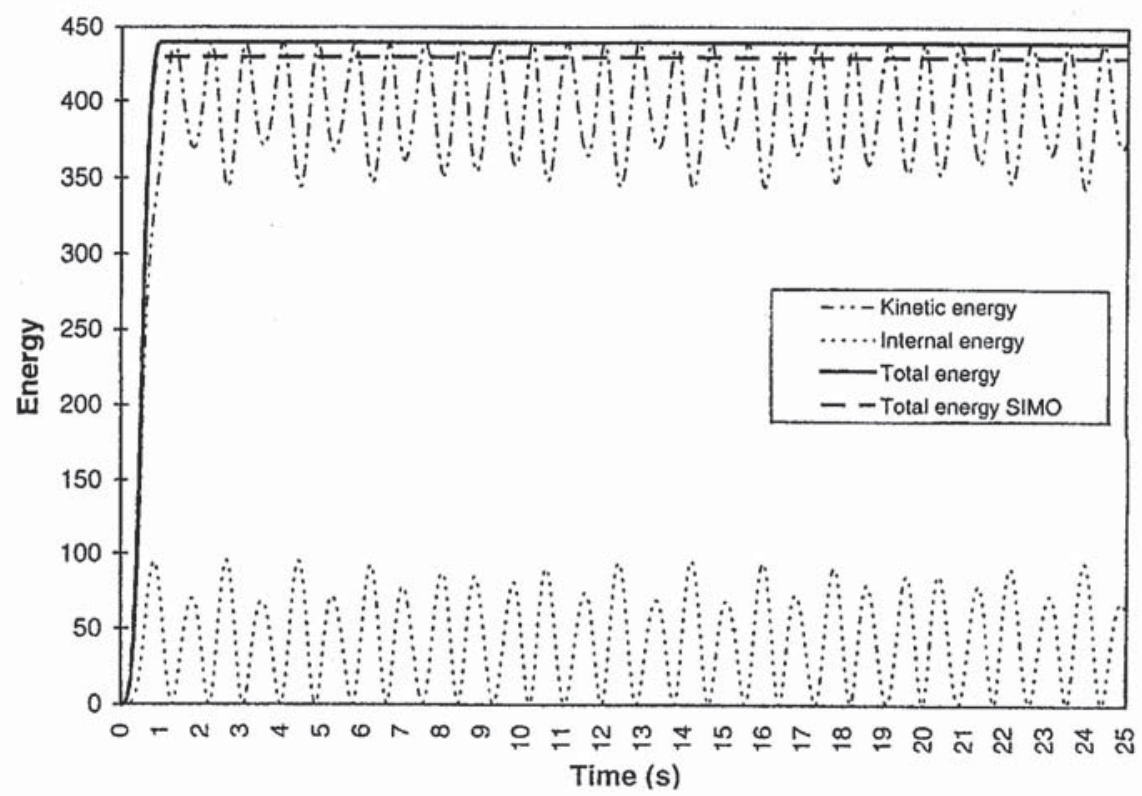

Figure 5. Short cylinder: time histories of kinetic, internal and total energies

thickness is $H=0.4$, while the height of the structure is $L=4 \cdot 60$. A time-dependent ring load is applied in $-Z$ direction on the upper edge of the cap by using 32 nodal forces, while the boundary conditions on the lower edge may be presented as: $U_{X} \neq 0, U_{Y} \neq 0, U_{Z}=0, \bar{T}^{1} \neq 0, \bar{T}^{2} \neq 0$, where $U_{A}$ represent displacements in global $A=X, Y, Z$ direction and $\bar{T}^{\alpha}$ are rotational parameters in local 


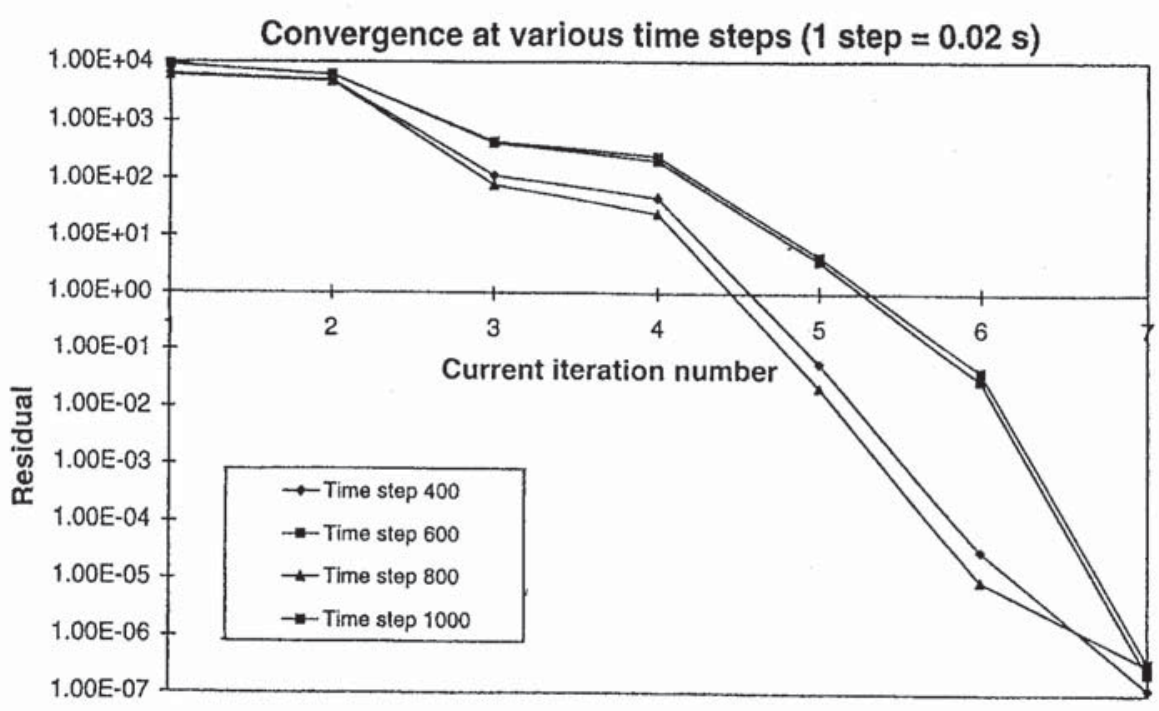

Figure 6. Short cylinder: convergence characteristics for various time steps
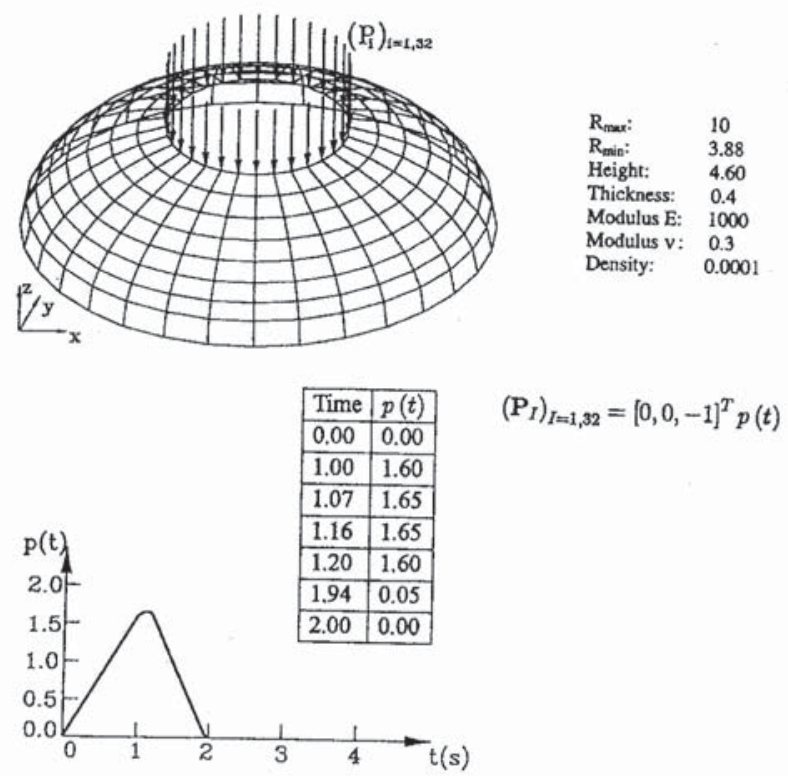

Figure 7. Spherical cup: geometry, material characteristics and loading conditions

$\mathbf{E}_{\alpha}$ directions (local coordinate systems are defined in accordance with the procedure described in Reference 21). The value and time history of the loading impulse is given in Figure 7. The response of the shell is traced up to $4.5 \mathrm{~s}$. The length of the step size is $\Delta t=0.0015 \mathrm{~s}$, which results in total number of 3000 time steps. The value of the ring load is such, that the dynamic snap-through occurs. The deformation process is evident from Figure 8 , where eight deformed 


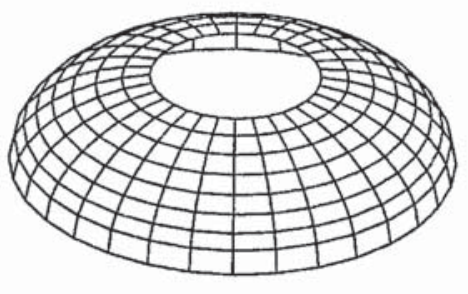

$t=0.15$

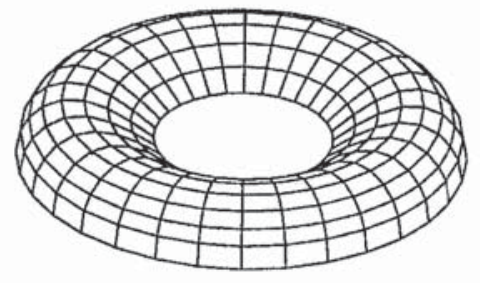

$\mathbf{t}=0.45$

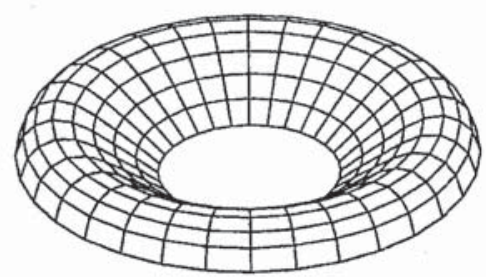

$t=0.75$

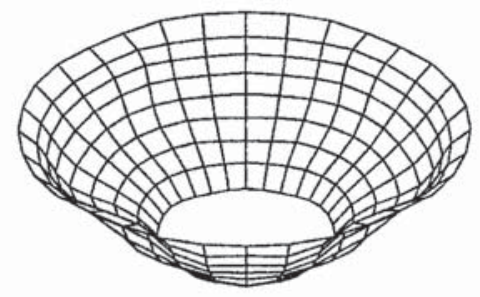

$t=1.05$

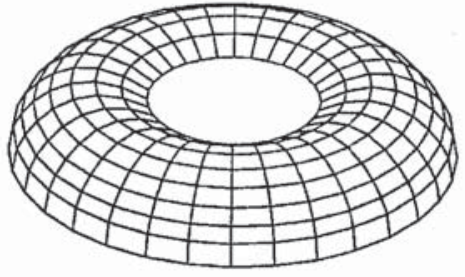

$t=0.30$

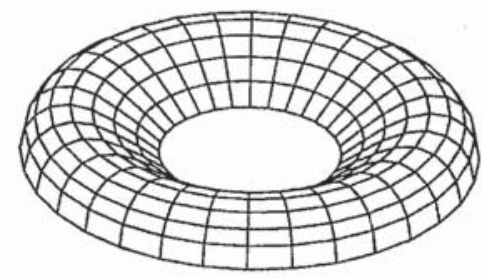

$t=0.60$

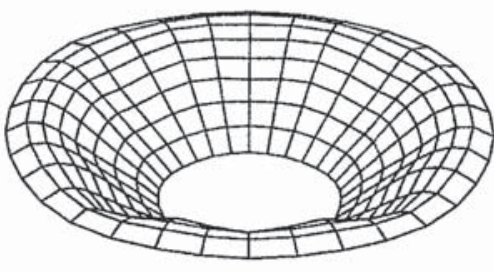

$t=0.90$

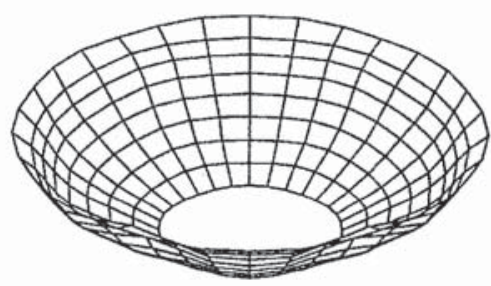

$t=1.20$

Figure 8. Spherical cup: sequence of deformed finite element meshes in the time interval $[0 \cdot 15,1 \cdot 20] \mathrm{s}$ 


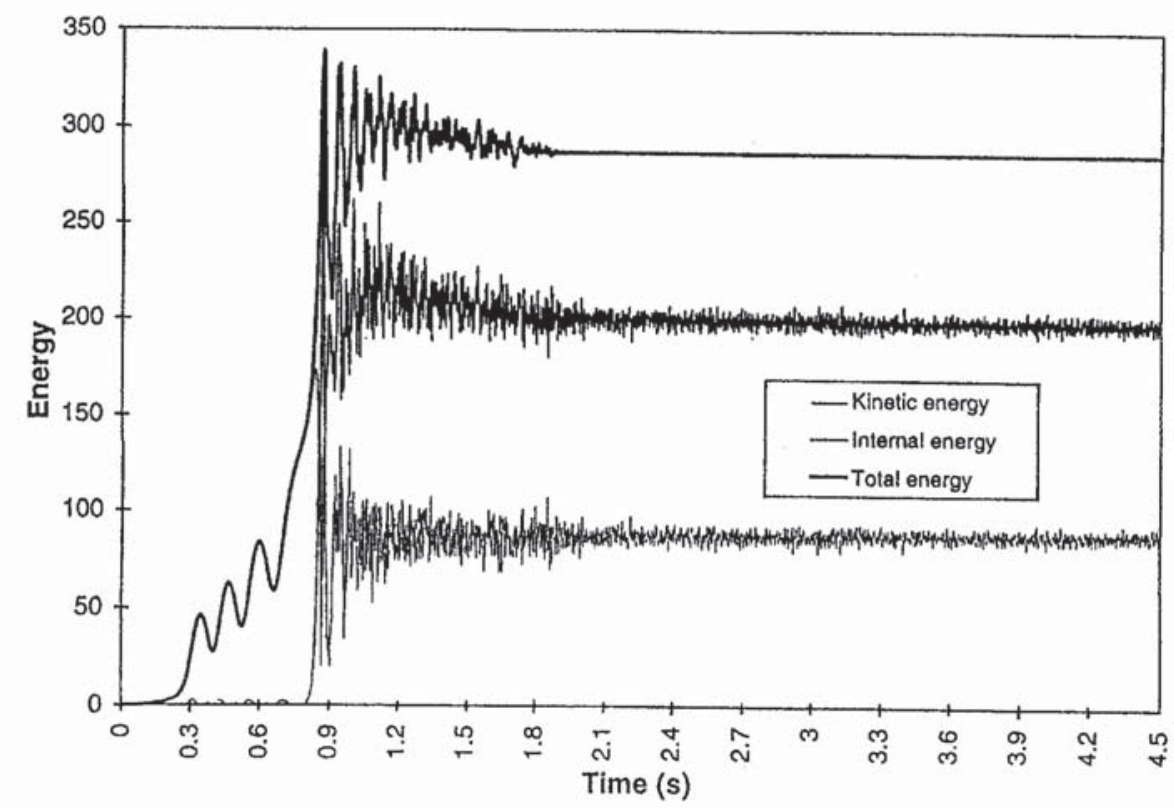

Figure 9. Spherical cup: time histories of kinetic, internal and total energies

finite element meshes are presented in the time interval $[0.15,1.20] \mathrm{s}$ in the sequence of $0.15 \mathrm{~s}$. In this example the total linear and the total angular momenta are not constant after the impulsive loading vanishes because of the non-zero boundary conditions applied, whereas the total energy is exactly conserved, as shown in Figure 9. It can be seen from Figure 9, that until approximately $t=0.8 \mathrm{~s}$ the kinetic energy of the system is practically zero. The snap-through occurs between the time instants $t=0.75 \mathrm{~s}$ and $t=0.90 \mathrm{~s}$. This results in a sudden increase of the kinetic energy. Very short-wave oscillations may be noticed in the time histories of the internal, external and total energy of the system in the time period $[0.8,2 \cdot 0] \mathrm{s}$. After the load is removed, the oscillations of the kinetic and internal energy are still present, while the total energy remains perfectly constant. It is interesting to note, that in the time history of the total energy oscillations are observed, i.e. the applied external loads produce the effect of dumping, which can be explained as follows. The total energy in the present case equals to

$$
E_{\text {tot }}(t)=\int_{t_{0}}^{t} \sum_{I=1}^{32} \mathbf{P}_{I}(\tau) \cdot \mathbf{u}_{I}(\tau) \mathrm{d} \tau=\int_{t_{0}}^{t} A(\tau) \mathrm{d} \tau
$$

with nodal forces and nodal displacements related to 32 nodes of the upper edge of the cap. The total energy at time instant $t$ is increasing if $A(t)>0$ and decreasing, exhibiting the effect of dumping, if $A(t)<0$. Although of some interest for this example, issues related to the dynamic stability are not the objective of this work and will not be further detailed. For the discussion on the non-linear dynamic stability, see e.g. Reference 22.

Example 6.3: Symmetric motion of a cylinder under two pinching forces. Geometry characteristics, material parameters, finite element mesh and loading conditions for this example are given in Figure 10. The shell is subjected to impulsive loading, represented by two symmetric forces, 


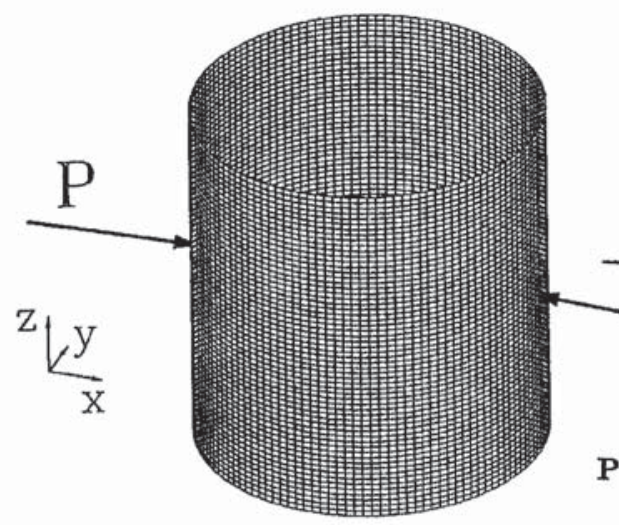

R: $\quad 300$

Height: $\quad 600$

Thickness: $\quad 3.0$

Modulus E: $\quad 3000$

Modulus v: 0.3

Density: $\quad 0.00008$

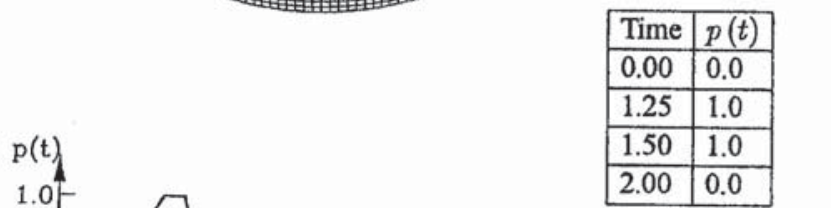

(b)

$t(s)$

Figure 10. Pynched cylinder: geometry, material characteristics and loading conditions

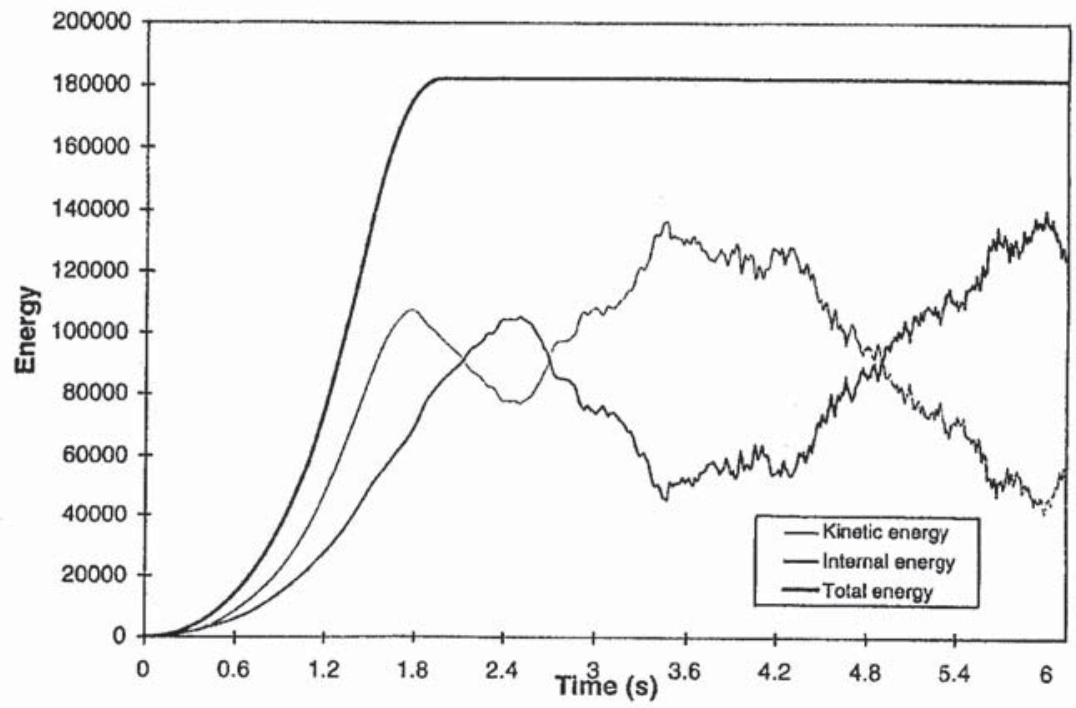

Figure 11. Pynched cylinder: time histories of kinetic, internal and total energies 

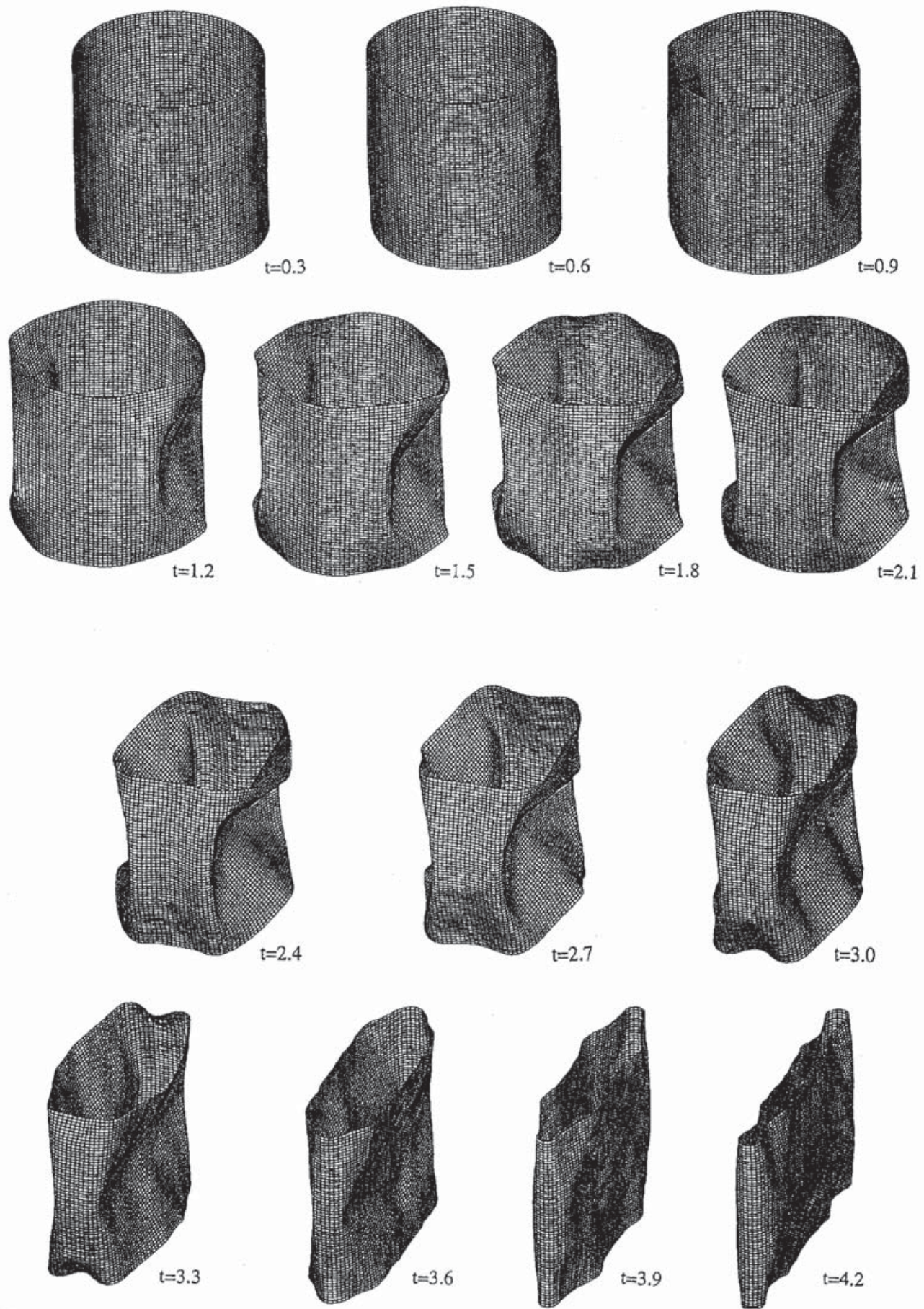

Figure 12. Pynched cylinder: sequence of 14 deformed finite element meshes in the time interval $[0.3,4 \cdot 2] \mathrm{s}$ 

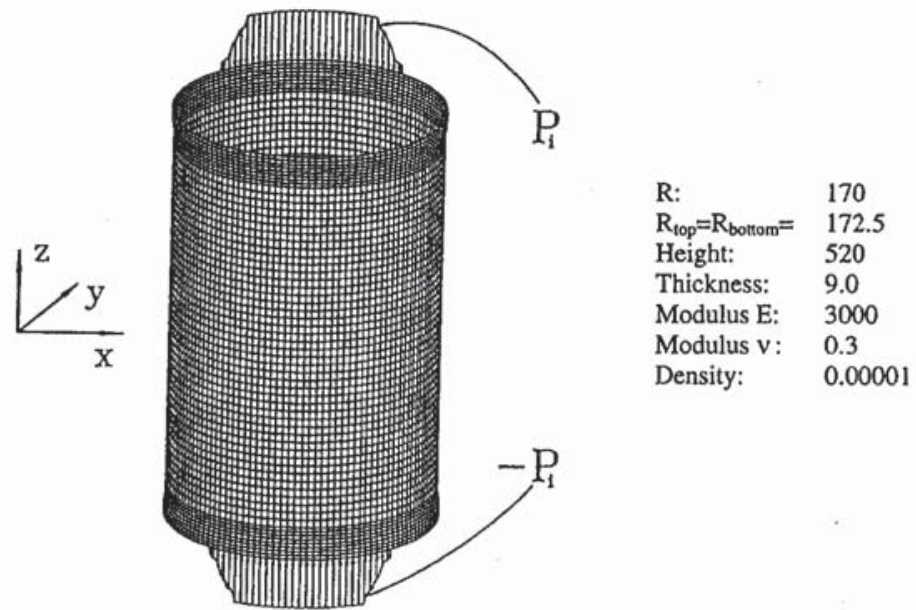

$\left(\mathbf{P}_{I}\right)_{I=1,128}=[0,0,-7400]^{T} p(t)$

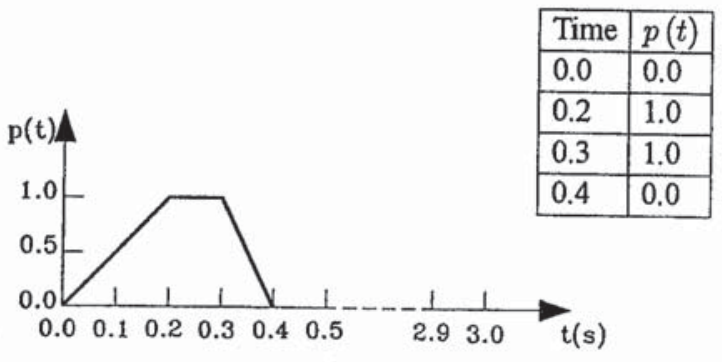

Figure 13. Axially loaded cylinder: geometry, material characteristics and loading conditions

acting on its middle cross-section. The time history of the acting loads is given in Figure 10. The initial conditions at $t=0$ are equal to zero. Only one-eighth of the cylinder is discretized with the finite element mesh. The motion of the structure is thus restricted by the boundary conditions for displacements and rotational parameters at the lines of the symmetry. The total linear and the total angular momentum are therefore not preserved after the two pinching forces vanish. The response is computed for $6.15 \mathrm{~s}$, resulting into 2050 time steps with $\Delta t=0.003 \mathrm{~s}$. The variation of kinetic, internal and the total energy of the system with respect to time is depicted in Figure 11. It can be observed that the main periods of internal and kinetic energy are very large and disturbed with the short-wave oscillations. In Figure 12 the deformed finite element meshes at various time steps in the period $[0 \cdot 3,4 \cdot 2] \mathrm{s}$ are presented. The somehow unusual mode of response is due to the adopted symmetry conditions, which restrict the motion of the shell at (two) planes of the symmetry to be strictly vertical and horizontal. As a result, the deformed shapes presented in Figure 12 are obtained. The undamped dynamic response after the second is periodical with the predominant motion in the global $X Y$ plane. The obtained convergence characteristics exhibit quadratic rate of asymptotic convergence and the maximum number of four iterations is needed at each time step. 

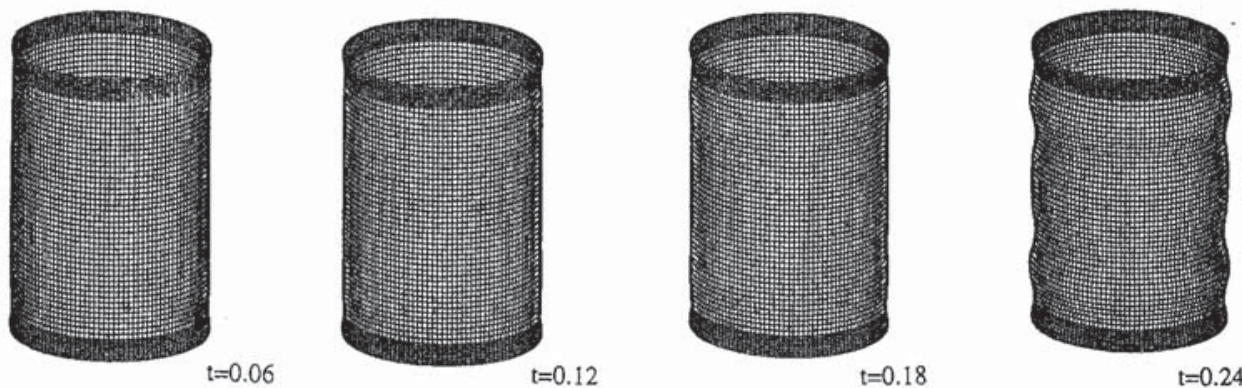

$t=0.30$

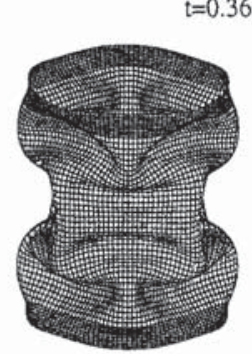

$t=0.42$
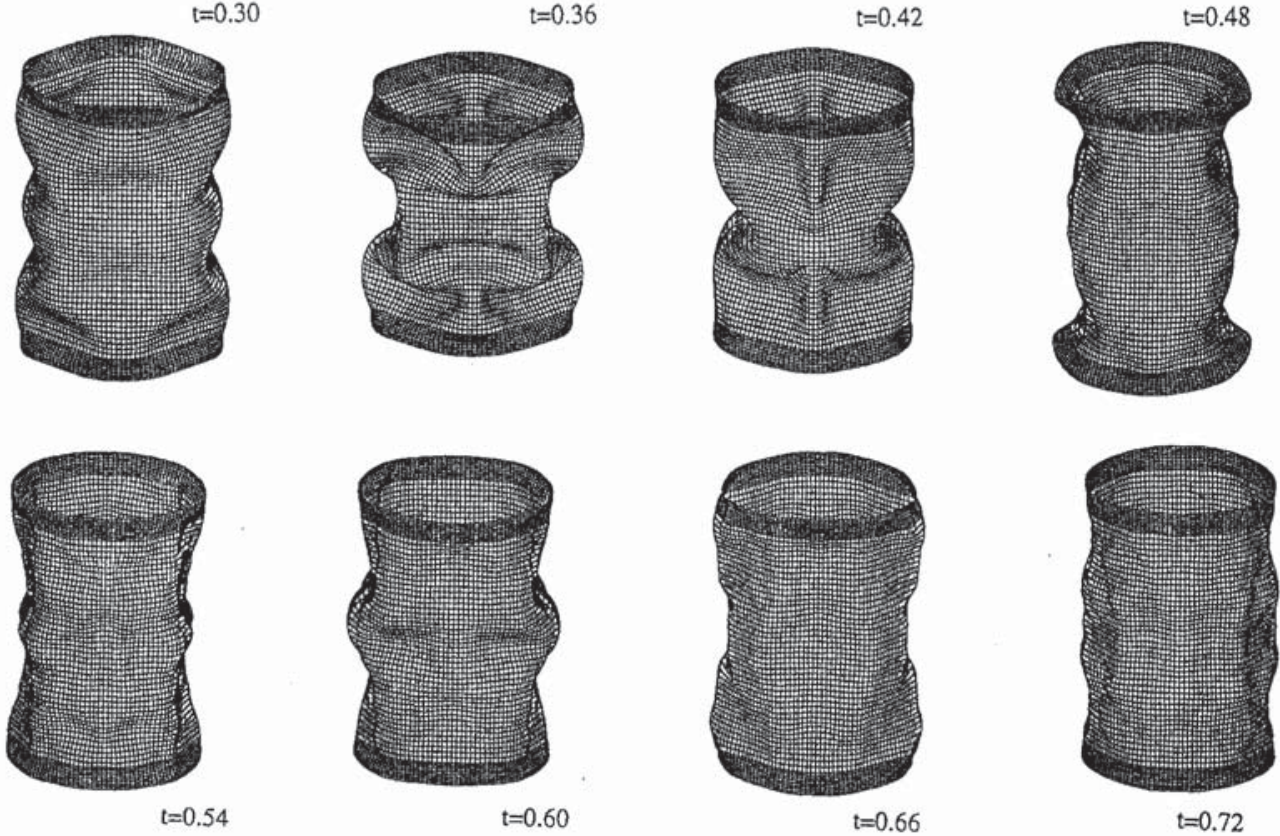

$t=0.54$

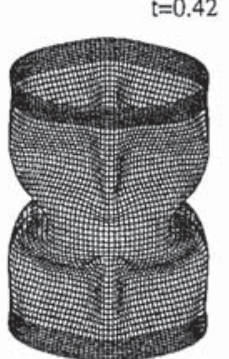

$\mathrm{t}=0.72$

$t=0.78$

$t=0.84$

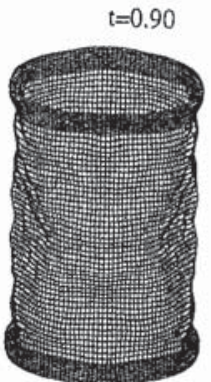

$t=0.96$
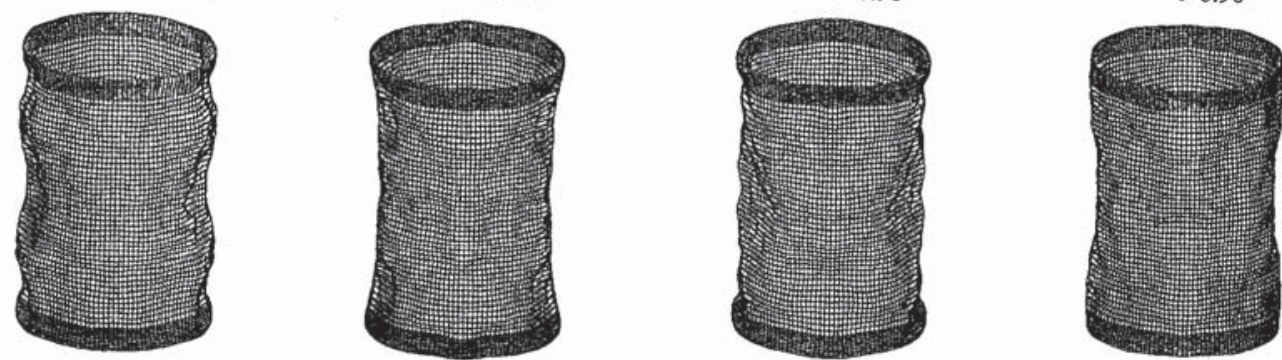

Figure 14. Axially loaded cylinder: sequence of 16 deformed finite element meshes 


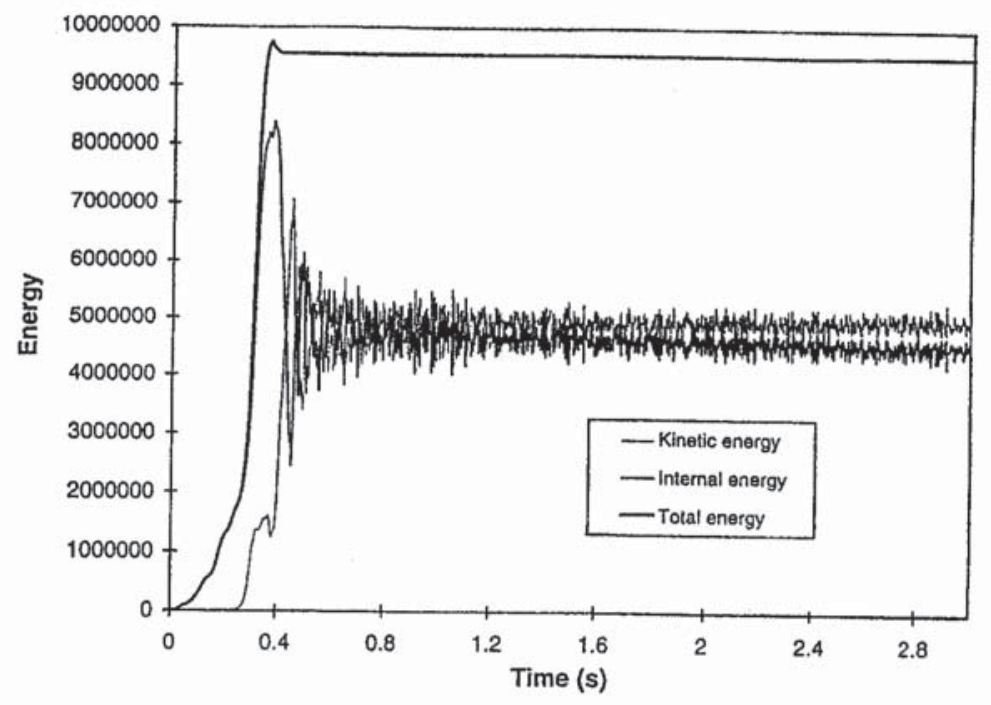

Figure 15. Axially loaded cylinder: time histories of kinetic, internal and total energies

Example 6.4: Free cylinder subjected to impulsive axial loading. The geometry, material characteristics, loading conditions and finite element mesh are presented in Figure 13. Radius of the cylinder, $R=170$, changes at the regions close to both ends being $R=172.5$ at the top and bottom ends. Time history of applied loads is given in Figure 13. The initial conditions at $t=0$ are set to zero. The response of the shell is traced up to $3.0 \mathrm{~s}$. A sequence of deformed finite element meshes in the time interval $[0.06,0.96] \mathrm{s}$ is presented in Figure 14 with the difference between two subsequent shapes to be $0.06 \mathrm{~s}$. Time histories of kinetic, potential and total energies are presented in Figure 15. Short-wave oscillations of kinetic and internal energy can be observed after $0.4 \mathrm{~s}$, while the total energy of the shell remains constant. The effect of dumping, discussed in Example 6.2 is also observed in the present example. In Figure 14 the direction of the shell motion between time instants $t=0.36 \mathrm{~s}$ and $t=0.42 \mathrm{~s}$ may be clearly observed. Since it is reversal to the direction of the applied loads the effect of dumping is produced.

\section{CONCLUSIONS}

In this work a formulation for the simulation of non-linear dynamic response of thin elastic shells is presented. The main features of the model can be briefly summarized as follows:

(i) Full non-linear kinematics is employed accounting for large displacements and large rotations. The motion of the shell director field is described by a singularity-free rotation vector formulation. The small strain hyperelastic elastic constitutive relations are assumed. Variation of the metrics across the shell thickness is neglected. First two strain tensors are taken into account.

(ii) The adopted time-stepping algorithm ${ }^{2,3,7-11}$ preserves fundamental constants of the unconstrained and unforced motion. For the Hamiltonian case it conserves the total energy. This is accomplished by evaluation of the weak form of the initial boundary value problem at the 
middle of the time interval $\left[t_{n}, t_{n+1}\right]$ and by defining the algorithmic stress resultants in a way that the total energy of the system is conserved.

(iii) The main features of the implementation of the energy-momentum conserving algorithm may be presented as follows: The algorithmic strains, used when defining the energy conserving form of algorithmic stress resultants, are evaluated for the quadratic stored energy function as an average of strains at time instants $t_{n}$ and $t_{n+1}$. The variations of the strains associated with intermediate configuration at time instant $t_{n+1 / 2}$ are obtained by strain-displacement operator, which is an average of strain-displacements operators at time instants $t_{n}$ and $t_{n+1}$. The update of rotational matrices at time instants $t_{n+1 / 2}$ and $t_{n+1}$ is performed by using an exponential mapping formulation.

(iv) Consistent linearisation of the fully discrete weak form of the initial boundary value problem leading to the Newton-Raphson numerical procedure is performed. The consistent 'mass' matrix (dynamic contribution to the tangent stiffness matrix) is generally non-symmetric and configuration dependent, since the time-dependent rotational matrices are involved. Non-symmetry is also present in the material and geometric (bending and transverse shear) parts of the tangent stiffness matrix.

(v) When the structural response is related to higher modes, e.g. when the structure is subjected to high impulsive loadings or when snap-through occurs, the adopted energy-momentum method demands relatively small time steps. Further research is needed for the complete insight into this problem.

A range of numerical example is chosen to illustrate the above points. The present approach has shown robust performance in the series of difficult tests.

The future work will be directed towards the inclusion of some form of dissipation into the constitutive description. This is necessary in order to perform more complete simulation of the dynamic phenomena.

\section{ACKNOWLEDGEMENTS}

The work has been financially supported from the University of Padua and the Ministry of Science and Technology of Slovenia. This support is gratefully acknowledged.

\section{REFERENCES}

1. Y. Basar and Y. Ding, 'Theory and finite element formulation for shell structures undergoing finite rotations', in G. Z. Voyiadjis and D. Karamanlidis (eds.), Advances in the Theory of Plates and Shells, Elsevier Science Publ.,
Amsterdam, 1990.

2. J. C. Simo, M. S. Rifai and D. D. Fox, 'On stress resultant geometrically exact shell model. Part VI. Conserving algorithms for non-linear dynamics', Int. J. Numer. Meth. Engng., 34, 117-164 (1992).

3. J. C. Simo and N. Tarnow, 'A new energy and momentum conserving algorithm for the non-linear dynamics of shells', Int. J. Numer. Meth. Engng., 37, 2527-2549 (1994).

4. J. C. Simo and D. D. Fox, 'On a stress resultant geometrically exact shell model. Part I. Formulation and optimal parametrization', Comput. Meth. Appl. Mech. Engng., 72, 267-304 (1989).

5. J. Stuelpnagel, 'On the parametrization of the three-dimensional rotation group', SIAM Rev., 6, 422-430 (1964).

6. T. J. R. Hughes, The finite element method, Prentice-Hall, Englewood Cliffs, N.J., 1984.

7. J. C. Simo and K. K. Wong, 'Unconditionally stable algorithms for rigid body dynamics that exactly preserve energy and momentum', Int. J. Numer. Meth. Engng., 31, 19-52 (1991).

8. J. C. Simo, N. Tarnow and K. K. Wong, 'Exact energy-momentum conserving algorithms and symplectic schemes for non-linear dynamics', Comput. Meth. Appl. Mech. Engng., 100, 63-116 (1992).

9. J. C. Simo and N. Tarnow, 'The discrete energy-momentum method. Conserving aigorithms for non-linear elastodynamics', Z. Agnew. Math. Phys., 43, 757-792 (1992). 
10. J. C. Simo, N. Tarnow and M. Doblare, 'On non-linear dynamics of three-dimensional rods: exact energy and momentum conserving algorithms', Int. J. Numer. Meth. Engng., 38, 1431-1474 (1995).

11. N. Tarnow, Energy and momentum conserving algorithms for Hamiltonian systems in the nonlinear dynamics of solids, Ph.D. Dissertation, Sudam Report No. 93-4, Department of Mechanical Engineering, Stanford University, Stanford California, 1993.

12. M. A. Crisfield and U. Galvanetto, 'Some unreliable finite element solutions for nonlinear dynamics', Proc. 5th Int. Conf. on Reliability of Finite Element Methods in Engineering, Vol. 10, 1994, 393-401.

13. U. Galvanetto and M. A. Crisfield, 'An energy-conserving co-rotational procedure for the dynamics of planar beam structures', Int. J. Numer. Meth. Engng., 39, 2265-2282, (1996).

14. D. Kuhl and E. Ramm, 'Constraint energy momentum algorithm and its application to nonlinear dynamics of shells', Comput. Meth. Appl. Mech. Engng., 136, 293-315 (1996).

15. E. N. Dvorkin and K.-J. Bathe, 'A continuum mechanics based four-node shell element for general non-linear analysis', Eng. Comput., 1, 77-88 (1984).

16. H. Parisch, 'An investigation of a finite rotation four node assumed strain shell element', Int. J. Numer. Meth. Engng., 31, 127-150 (1991).

17. M. Borri and C. Bottasso, 'A general framework for interpreting time finite element formulations', Comput. Mech., 13, 133-142 (1993).

18. B. Brank, Large Deformations of Materially Non-Linear Shells (in Slovenian), Dissertation, Faculty of Civil and Geodetic Engineering, University of Ljubljana, Ljubljana, 1994.

19. B. Brank, D. Perić and F. B. Damjanić, 'On large deformations of thin elasto-plastic shells: Implementation of a finite rotation model for quadrilateral shell element', Int. J. Numer. Meth. Engng., 40, 689-726 (1997).

20. B. Brank, D. Perić and F. B. Damjanić, 'On implementation of a non-linear four-node shell finite element for thin multilayered elastic shells', Comp. Mech., 16, 341-359 (1995).

21. J. A. Figuerias and D. R. J. Owen, 'Analysis of elasto-plastic and geometrically nonlinear anisotropic plates and shells', in E. Hinton and D. R. J. Owen, (eds.), Finite Element Software for Plates and Shells, Pineridge Press, Swansea, 1984 , pp. 235-326.

22. Y. Basar, C. Eller and W. B. Krätzig, 'Finite element procedures for the non-linear dynamic stability analysis of arbitrary shell structures', Comp. Mech. 6, 157-166 (1990). 